\title{
Article
}

\section{An Efficient Shortest Path Algorithm: Multi-Destinations in an Indoor Environment}

\author{
Mina Asaduzzaman ${ }^{1}(0)$, Tan Kim Geok ${ }^{2, *}$, Ferdous Hossain ${ }^{2, *}{ }^{\complement}$, Shohel Sayeed ${ }^{1}$, Azlan Abdaziz ${ }^{2}$, \\ Hin-Yong Wong ${ }^{3}$, C. P. Tso ${ }^{2}$, Sharif Ahmed ${ }^{2}$ and Md Ahsanul Bari ${ }^{1}$
}

1 Faculty of Information Science and Technology, Multimedia University, Melaka 75450, Malaysia; 1181402835@student.mmu.edu.my (M.A.); shohel.sayeed@mmu.edu.my (S.S.); 1141123630@student.mmu.edu.my (M.A.B.)

2 Faculty of Engineering and Technology, Multimedia University, Melaka 75450, Malaysia; azlan.abdaziz@mmu.edu.my (A.A.); cptso2014@gmail.com (C.P.T.); 1171402467@student.mmu.edu (S.A.)

3 Faculty of Engineering, Multimedia University, Cyberjaya 63100, Malaysia; hywong@mmu.edu.my

* Correspondence: kgtan@mmu.edu.my (T.K.G.); ferdous.mbstu.cse@gmail.com (F.H.); Tel.: +60-013-613-6138 (T.K.G.); +60-112-108-6919 (F.H.)

Citation: Asaduzzaman, M.; Geok, T.K.; Hossain, F.; Sayeed, S.; Abdaziz, A.; Wong, H.-Y.; Tso, C.P.; Ahmed, S.; Bari, M.A. An Efficient Shortest Path Algorithm: Multi-Destinations in an Indoor Environment. Symmetry 2021, 13, 421. https://doi.org/10.3390/ sym 13030421

Academic Editor: Peng-Yeng Yin

Received: 28 December 2020

Accepted: 31 January 2021

Published: 5 March 2021

Publisher's Note: MDPI stays neutral with regard to jurisdictional claims in published maps and institutional affiliations.

Copyright: (c) 2021 by the authors. Licensee MDPI, Basel, Switzerland. This article is an open access article distributed under the terms and conditions of the Creative Commons Attribution (CC BY) license (https:// creativecommons.org/licenses/by/ $4.0 /)$.

\begin{abstract}
The shortest path-searching with the minimal weight for multiple destinations is a crucial need in an indoor applications, especially in supermarkets, warehouses, libraries, etc. However, when it is used for multiple item searches, its weight becomes higher as it searches only the shortest path between the single sources to each destination item separately. If the conventional Dijkstra algorithm is modified to multi-destination mode then the weight is decreased, but the output path is not considered as the real shortest path among multiple destinations items. Our proposed algorithm is more efficient for finding the shortest path among multiple destination items with minimum weight, compared to the single source single destination and modified multi-destinations of Dijkstra's algorithm. In this research, our proposed method has been validated by real-world data as well as by simulated random solutions. Our advancement is more applicable in indoor environment applications based on multiple items or destinations searching.
\end{abstract}

Keywords: graph; multi-destination; road network; shortest path; indoor

\section{Introduction}

In computer science, finding the shortest path in the complex indoor environment is a fundamental issue [1]. The solution to this issue is to use a bi-directional graph or road network to find the path with the minimal summation of edge weights among all reachable paths from start to end nodes [2]. The bi-directional graph considered in this paper is either directed or undirected, finite, simple, as well as connected [3]. The definition of a single pair shortest path is a path that contains only one destination. In conventional problems, the distances among the nodes are ascertained. The goal of the conventional single-source SPP is to get the minimal cost path from the source node to the destination node. However, the fuzzy number technique can be utilized instead in precarious environments [4]. The problem arises when the graph contains more than one destination node [5]. The set of destination nodes is a subset of all network nodes and it becomes a multi-destination shortest path problem [6], which has many real-life applications, such as finding the shortest path among multi-destination items in supermarkets, warehouses, libraries, road networks [7], robotics industries [8], service compositions [9,10], and multicast routes [11]. The different techniques found in the literature are calculating either a multi-destination or single-pair shortest path to solve these issues [12]. $A^{*}$ is an ordinary BFS algorithm that depends on a heuristic function to demonstrate the search towards finding the shortest path from a source node to a destination node in a grid [13]. As of now, Dijkstra's is a renowned path searching algorithm that is mainly used for searching the shortest path from 
a single source to a single destination introduced by Edsger W. Dijkstra in 1956. A typical single-source single-destination, Dijkstra's algorithm, is used for single-pair shortest path problems [14]. It keeps up a set S of unraveled nodes, consisting of those hubs or nodes whose ultimate shortest path between the starting node verses has ascertained as well as labels $t(\mathrm{i})$, conserving the upward bound of the smallest pathway distance among $v_{\mathrm{S}}$ and $v_{\mathrm{i}}$ [15]. The node $v_{\mathrm{m}} \in V / S$ with the minimal $t(\mathrm{i})$ is continually chosen by the algorithm, adding $v_{\mathrm{m}}$ to $S$, as well as updating $t(\mathrm{i})$ as the node $v_{\mathrm{m}}[16]$ :

In the first step. Set $t\left(v_{\mathrm{s}}\right)=0$ and $t\left(v_{\mathrm{j}}\right)=w_{\mathrm{sj}}$.

For other nodes $S=\left\{v_{\mathrm{s}}\right\}, L=V / S$

In the second step.

Chose a hub $v_{\mathrm{m}}$ from $\mathrm{L}, t\left(v_{\mathrm{m}}\right)=\min _{v_{\mathrm{j}} \in L} t\left(v_{\mathrm{j}}\right)$,

if $t\left(v_{\mathrm{m}}\right)=1$, then stop, else move to third step.

In the third step set.

$$
S=S \mathrm{U}\left\{v_{\mathrm{m}}\right\}, L=L \backslash\left\{v_{\mathrm{m}}\right\}
$$

if $L=\varnothing$, then stop, else move to forth step.

In the fourth step.

For each $v_{\mathrm{j}} \in L$, update

$$
t\left(v_{\mathrm{j}}\right)=\min \left\{t\left(v_{\mathrm{j}}\right), t\left(v_{\mathrm{m}}\right)+w_{\mathrm{mj}}\right.
$$

Move to first step.

In the above steps, $V=\left\{v_{1}, v_{1}, \ldots, v_{\mathrm{n}}\right\}$ is the set of nodes, $S$ is the set of the source nodes, $\mathrm{L}$ is a list, and $W=w_{\mathrm{ij}}$ is the weight matrix. The above steps demonstrate the shortest path calculation following the steps of Dijkstra's algorithm and the final output is expressed in Equation (1).

The Modified Dijkstra's Multi-Source Multi-Destination (MDMSMD) algorithm is applied for the multi-destination shortest path problem [17]. For the single-source multidestination problem, the conventional Dijkstra's single-source single-destination (CDSSSD) algorithm can be used. Although, in this network, the starting point is one node and destinations are a set of multiple nodes as a source node (s) and multiple destination nodes $(w, x, y, z)$ as shown in Figure 1. As a result, it is necessary to calculate the shortest path between $\mathrm{s}$ to $\mathrm{w}, \mathrm{s}$ to $\mathrm{x}, \mathrm{s}$ to $\mathrm{y}$, and $\mathrm{s}$ to $\mathrm{z}$.

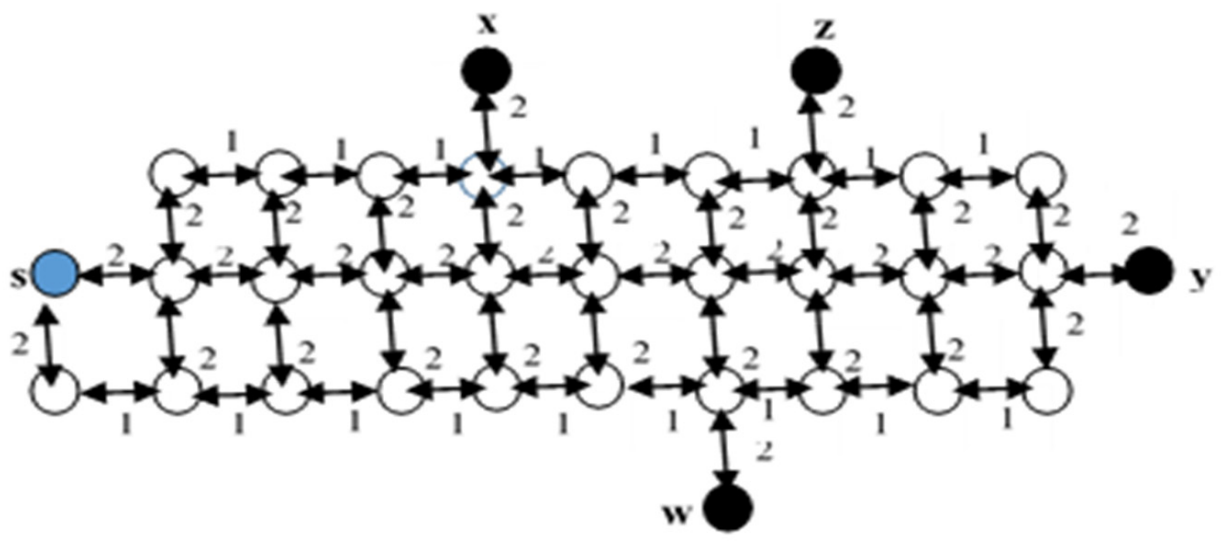

Figure 1. Bi-directional road network.

From the sample road network Figure 1, the steps of CDSSSD in Figure 2 is displayed. Figure $2 \mathrm{a}$ is the first step that calculates the shortest path between $\mathrm{s}$ and $\mathrm{w}$ from all accessible paths. Figure $2 b$ is the second step that calculates the shortest path between $\mathrm{s}$ and $\mathrm{x}$. Figure $2 \mathrm{c}$ shows the third step that calculates the shortest path between $\mathrm{s}$ and $\mathrm{y}$, Figure $2 d$ also shows the shortest path between $x$ and $z$. In Figure $2 e$, all the calculated shortest paths among $\mathrm{s}$ and the destination nodes $\mathrm{w}, \mathrm{x}, \mathrm{y}$, and $\mathrm{z}$ [18]. Here, the possible number of shortest paths is four. However, the number of paths can be converted to a single 
path from a source to the last destination node of a set of destination nodes by applying the MDMSMD. For example, a source node is s and the destination nodes are $w, x, y$, and $\mathrm{z}$ as per Figure 1. Hence, the shortest paths are between $\mathrm{s}$ and $\mathrm{w}, \mathrm{s}$ and $\mathrm{x}, \mathrm{s}$ and $\mathrm{y}$, and $\mathrm{s}$ and $\mathrm{z}$. The path of MDMSMD is $\mathrm{s} \rightarrow \mathrm{w} \rightarrow \mathrm{x} \rightarrow \mathrm{y} \rightarrow \mathrm{z}$ and has been expressed in Figure 3 . In Figure 3a-e are respectively shown the shortest path between $s \rightarrow \mathrm{w}$ is $10, \mathrm{w} \rightarrow \mathrm{x}$ is 9 , $\mathrm{x} \rightarrow \mathrm{y}$ is $11, \mathrm{y} \rightarrow \mathrm{z}$ is 8 and $\mathrm{s} \rightarrow \mathrm{w} \rightarrow \mathrm{x} \rightarrow \mathrm{y} \rightarrow \mathrm{z}$ is 38 . The minimum summation of edge weights of MDMSMD is less than CDSSSD. The MDMSMD solved the multi-destination path problem, with some limitations [19]. The MDMSMD calculates the shortest path from each source to each destination node, the previous destination node is the next source node, and calculates the shortest path from the next source to the next destination node until the last destination node. This algorithm can only calculate the shortest path between one pair at a time [20], it cannot calculate the shortest path between a source node and a set of destination nodes at the same time.

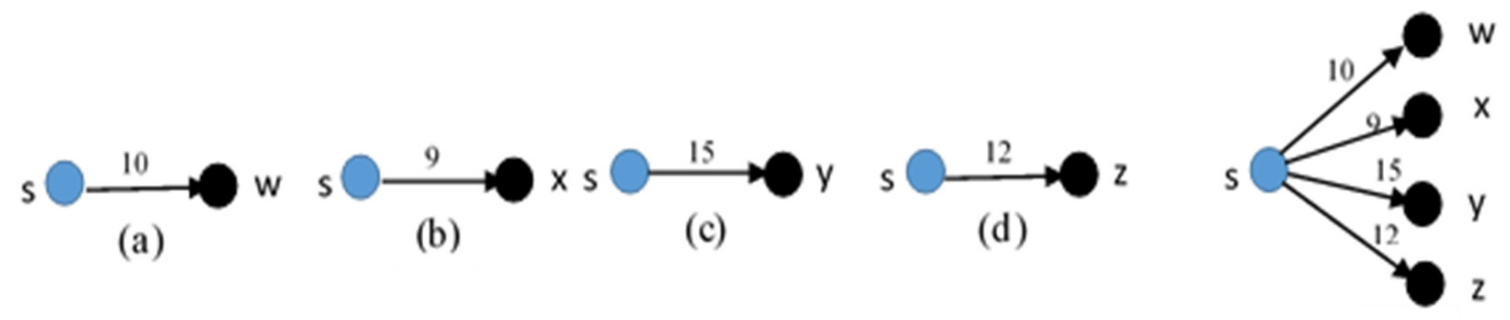

(e)

Figure 2. CDSSSD.

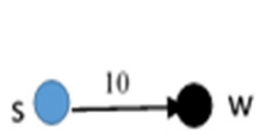

(a)

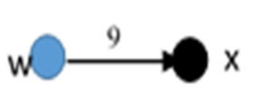

(b)

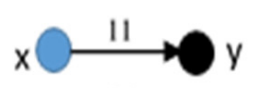

(c)

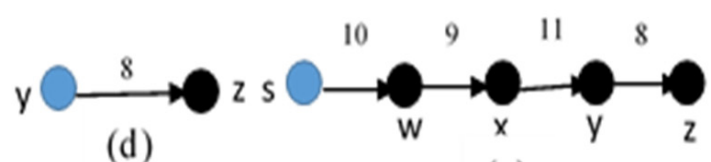

(e)

Figure 3. MDMSMD.

The objective of this research is to develop an efficient algorithm for searching the smallest path between a given source and the multi-destination nodes. The name of this proposed algorithm is the efficient algorithm for multi-destination shortest path problem (EAMDSP) - it accentuates the searching area and enhances the algorithm performance. The new concept is based on considering the outcome of the edge weights of the input graph on searching speed to acquire the goal. Using this concept, the EAMDSP finds the nearest destination node among the multiple destination nodes from the source node. After getting the first nearest destination node, it becomes the next source node and again calculates the next nearest destination node among the remaining multiple destination nodes until the last or rest destination node. Finally, the shortest path between a source node and the last destination node that decreases the summation of weights is achieved, which enhances the speed of searching for the desired shortest path. The proposed algorithm acquires the benefits of simplicity as well as the effectiveness of the computations. The outcomes of this algorithm using a real dataset and the simulated random solution will show that the proposed algorithm is better than the existing algorithm.

In this article, the following aims are discussed: Section 2, for the multi-destination shortest path problem, the existing method and solution have been reviewed as well as discussed. We propose an algorithm for searching the multi-destination shortest path in indoor environments in Section 3. In Section 4, the performance of the proposed algorithm is compared to the existing method and the conclusion is in Section 5. 


\section{Related Work}

Nowadays, many researchers are working on graphical solutions and pathfinding to resolve crucial application issues [21-27]. Dijkstra's algorithm is used for finding the solution to the shortest path problem in multi-destination nodes [28]. The Fibonacci heap [29,30] has been utilized in the multi-destination Dijkstra's algorithm and is considered an innovative improvement. Searching the shortest paths by the node combination method is introduced by Lu et al. [31] by continually integrating with the source node's contiguous neighbors. However, multi-destination Dijkstra's algorithm is faster than their method. A quicker strategy with a small number of positive integer weights is introduced by Orlin et al. [32]. Thorup [33,34] invents an effective algorithm to solve the limitation of the Orlin et al. method which is an integral edge weight in every direction, as well as an undirected graph. In reality, no method has been utilized as an input of both undirected and directed graphs [35]. The breadth-first search, as well as the Bellman-Ford algorithm, are proposed respectively in the literature, while the graph contains all equal edge positive/negative weights. Searching the shortest path via a topological sequence [16] in linear time is feasible for a directed graph. An algorithm is introduced by Xu et al. [36] to effectively give a solution to the issue, particularly for infrequent networks.

In an article by [37], calculating the path as a usual job, so for every application [38,39] accelerate procedures perform based on the kind of data. A multi-destination feature of Dijkstra's algorithm is becoming compatible with recent applications [40,41]. By utilizing an amended version of multiple destinations, Dijkstra's algorithm is able to solve the bi-objective multi-destination shortest path problem in the pathway network which is introduced by Ticha et al. [7]. Their searching technique at each repetition of a process is to choose some parts of the path for a non-dominated path to arrive at one of the destination nodes. In the field of time schedule networks, Jin et al. [42] modified the multi-destination method for searching for the shortest path. First, they developed a path calculation method, then added it to the modified algorithm. Mainly, the shortest path calculation depends on the network constraints on its edges. By considering both node weights and edge weights, the multi-destination Dijkstra's method enhancement is proposed by Ananta et al. [43]. For the software-defined networks, this method transmits data packets to all destination nodes. The limitations of multi-destination Dijkstra's algorithm have been discussed in Section 1. This section also discussed the related algorithms, but they have some limitations such as one is designed only for a directed graph or only works on positive edge weight and another contains both positive and negative edge weight. The proposed algorithm has been designed for removing their short-comings and can perform on a bi-directional graph or road network, as well as finding the shortest path from the starting node to the multiple destination nodes, as is required in indoor environments.

\section{Algorithms}

Algorithm 1 (CDSSSD) and Algorithm 2 (MDMSMD) are used as reference algorithms to compare the performance of our proposed Algorithm 3. The steps of CDSSSD, MDMSMD, and EAMDSP are provided in this section to demonstrate a clear overview. 


\subsection{Algorithm: CDSSSD}

\begin{tabular}{l}
\hline Algorithm 1 Input: Graph: G (V, E, W), source node: S, set of multiple destination nodes: $\mathrm{T}$ \\
Output: Paths from source to destination nodes \\
\hline
\end{tabular}

1. Declare: $\mathrm{L}$

2. For $(i=0 ; \mathrm{I}<$ T.Length; $\mathrm{i}++)$

$3 . \quad$ Declare: nodes_path

4. $\mathrm{n}:=$ G.GetLength $(0)$;

5. $\quad$ distance: = new int[n];

6. For (int $\mathrm{k}=0 ; \mathrm{k}<\mathrm{n} ; \mathrm{i}++$ )

7. distance[i]: = int.MaxValue;

8. $\quad$ End For

9. distance[S]: $=0$;

10. used: = new bool[n];

11. Previous: = new int?[n];

12. While (true)

13.

14.

minDistance: $=$ int.MaxValue;

minNode: $=0$;

For (int $\mathrm{m}=0 ; \mathrm{m}<\mathrm{n} ; \mathrm{m}++$ )

If (!used[m] \&\& minDistance $>$ distance[m])

minDistance: $=$ distance $[\mathrm{m}]$;

minNode: $=\mathrm{m}$;

End If

End For

If $($ minDistance $==$ int.MaxValue)

break;

End If

used[minNode]: = true;

For (int $1=0 ; 1<\mathrm{n} ; 1++$ )

If $(\mathrm{G}[$ minNode, 1$]>0)$

shortestToMinNode: $=$ distance[minNode];

distanceToNextNode: $=$ G[minNode, 1$]$;

totalDistance: $=$ shortestToMinNode + distanceToNextNode;

If (totalDistance < distance[1])

distance[1]: = totalDistance;

previous[1]: = minNode;

End If

End If

End For

End While

If (distance[T[i]] :== int.MaxValue)

return null;

End If

currentNode: $=\mathrm{T}[\mathrm{i}]$;

While (currentNode $!=$ null)

Add nodes_path->col: = currentNode.Value;

currentNode: $=$ previous[currentNode. Value];

End While

col1: $=\mathrm{S}$;

For $(j=0 ; j<$ nodes_path.Length; $j++)$

Add L->col: = nodes_path[j]

col1: = nodes_path[j];

End For

49.

50.

End For

51. Return L; 


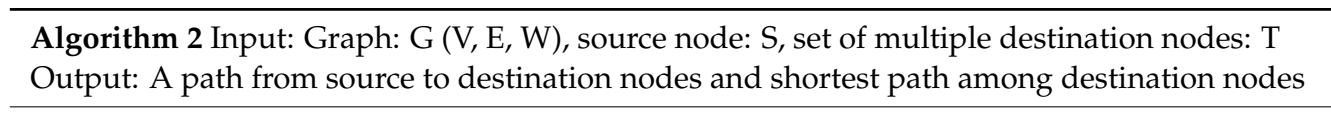

1. Declare: $\mathrm{L}$

2. $\quad$ For $(\mathrm{i}=0 ; \mathrm{I}<$ T.Length; $\mathrm{i}++)$

3. Declare: nodes_path

4. $\mathrm{n}:=$ G.GetLength $(0)$;

5. $\quad$ distance: $=$ new int[n];

6. For (int $\mathrm{k}=0 ; \mathrm{k}<\mathrm{n} ; \mathrm{i}++$ )

7. distance[i]: = int.MaxValue;

8. $\quad$ End For

9. $\quad$ distance[S]: $=0$;

10. used: = new bool[n];

11. Previous: = new int?[n];

12. While (true)

13.

14.

15.

16.

17.

18.

19.

20.

21.

22.

23.

24.

25.

26.

27. 


\subsection{The Proposed EAMDSP}

In algorithm 3, we consider that $\mathrm{G}=(\mathrm{V}, \mathrm{E}, \mathrm{W})$ is a graph containing $\mathrm{N}$ nodes (vertices), a set of edges, and a set of weights. The vertex set $\mathrm{V}$ has $n=|\mathrm{V}|$ collection of nodes, $\mathrm{E}$ contains $m=|E|$ and is the collection of edges, as well as $\mathrm{W}$, the collection of weights.

Let $W_{e} \in W$ be the weight of edge $e=(u, v) \in E$ as $u \rightarrow v$ for some $u, v \in V$.

The objective is to find a collection of shortest paths among vertices $\mathrm{s} \in \mathrm{V}$ as an assigned origin node to the collection of multi-destination nodes $d_{\mathrm{i}} \in \mathrm{D} \subseteq \mathrm{V}, i \in I=\{1$, $2, \ldots,|\mathrm{D}|\}$. For instance, $w_{\text {path }\left(s, d_{i}\right)}=\sum_{j}^{\mid \text {path }\left(s, d_{i}\right) \mid} w_{e j}$ where the path $\left(s, d_{i}\right)$ is the weight of path and $p d_{i}(i \in I)$ is the shortest path containing the minimal weight of $w_{\text {path }\left(s, d_{i}\right)}$ among all accessible paths from $s$ to $d_{i}$. Here, if there is no path from $s$ to $d_{i}$ then $\delta p d_{i}=\infty$ else $\delta p d_{i}=\delta$ path $\left(s, d_{i}\right)=\min \left\{w_{\text {path }(s, d i)}\right\}$. Figure 1 explains the details of this scenario.

The proposed algorithm, EAMDSP, provides the shortest path from source node $s \in V$ to all destination nodes or multi-destination nodes $d_{i} \in T \subseteq V, i \in I$ in the graph $\mathrm{G}=(\mathrm{V}, \mathrm{E}, \mathrm{W})$.

Firstly, find the nearest destination node from the set of destination nodes by calculating the shortest path among all possible paths using Dijkstra's single source-single destination algorithm.

Secondly, the nearest destination node from the source node is identified by minimum visited nodes and a minimum sum of weights between the source and each destination node.

Thirdly, after getting the first nearest destination node, it is considered as the next source node and starts the calculation of the nearest destination node from the rest of the destination nodes. This procedure is repeated until the last rest destination node. Getting the visited nodes from the source to the first nearest node combines the visited nodes, and goes for the next sources and continues until the last shortest path. The minimum sum of weights also adds as the above-visited nodes. Finally, the path that is found from the source to the last destination is the shortest path between the source to multi-destination nodes with minimum visited nodes and the minimal sum of weights.

For instance, in Figure 1, the source node is s and destination nodes are sequentially w, x, y, and z. In Figure 4, the steps of EAMDSP have been demonstrated in Figure 1, with a sample bi-directional road network with weights and nodes. In step (a) of Figure 4, the sum of weights calculated from $\mathrm{s}$ to $\mathrm{w}$ is $10, \mathrm{~s}$ to $\mathrm{x}$ is 9 , $\mathrm{s}$ to $\mathrm{y}$ is 15 , and $\mathrm{s}$ to $\mathrm{z}$ is 12 , and are found after calculating the shortest path among possible accessible paths.

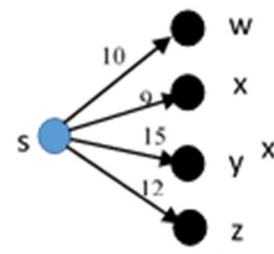

(a)

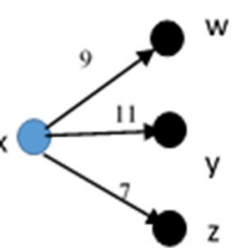

(b)

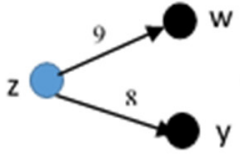

(c)

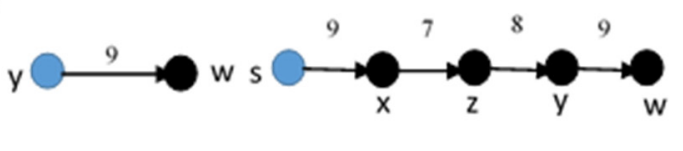

(d)

(e)

Figure 4. Graphical representation of EAMDSP.

First, each shortest path is $\mathrm{s} \rightarrow \mathrm{x}$ then $\mathrm{x}$ becomes the source node in the next step (b) of Figure 4. Similarly, the rest ( $w, y, z)$ are destination nodes, and again calculation on the sum of the weights from $x$ to $w$ is $9, x$ to $y$ is 11 , and $x$ to $z$ is 7 . Secondly, the shortest path is $\mathrm{x} \rightarrow \mathrm{z}$ and combined with the minimum visited nodes between $\mathrm{x} \rightarrow \mathrm{z}$. Furthermore, the previous minimum visited nodes between $\mathrm{s}>\mathrm{x}$ are combined with the minimum sum of weights from $\mathrm{s} \rightarrow \mathrm{x}$ to $\mathrm{x} \rightarrow \mathrm{z}$.

Now in step (c) of Figure 4, $\mathrm{z}$ is the source and $\mathrm{w}$ and $\mathrm{y}$ are destination nodes. Again, the calculation is done on the shortest path between $z \rightarrow y$ and $z \rightarrow w$. The minimum sum of weights between $\mathrm{z} \rightarrow \mathrm{y}$ is eight and $\mathrm{z} \rightarrow \mathrm{w}$ is nine. the shortest path is $\mathrm{z} \rightarrow \mathrm{y}$ and combined with the minimum visited nodes between $z>y$. The previous minimum visited nodes between $\mathrm{z}>\mathrm{y}$ are combined with the minimum sum of weights from $\mathrm{s}>\mathrm{x}>\mathrm{z}$ to $\mathrm{z}>\mathrm{y}$. 
In step (d) of Figure 4, calculate the shortest path between $y$ to $\mathrm{w}$ and get the sum of the weight values. Finally, the shortest path is $\mathrm{s} \rightarrow \mathrm{x} \rightarrow \mathrm{z} \rightarrow \mathrm{y} \rightarrow \mathrm{w}$, and the total sum of weights is 33 .

The MDMSMD working has been discussed in Section 1. The EAMDSP calculates the nearest destination node from the set of destination nodes that is the main difference from the MDMSMD. In the above example, the MDMSMD path will be $\mathrm{s} \rightarrow \mathrm{w} \rightarrow \mathrm{x}-\mathrm{y}-\mathrm{z}$ and the total sum of weight is 38. The CDSSSD total sum of weights is 46 from the above example. The number of total visited nodes and the sum of minimum weights of EAMDSP is less than the MDMSMD and the CDSSSD. As a result, EAMDSP is more efficient than MDMSMD and CDSSSD.

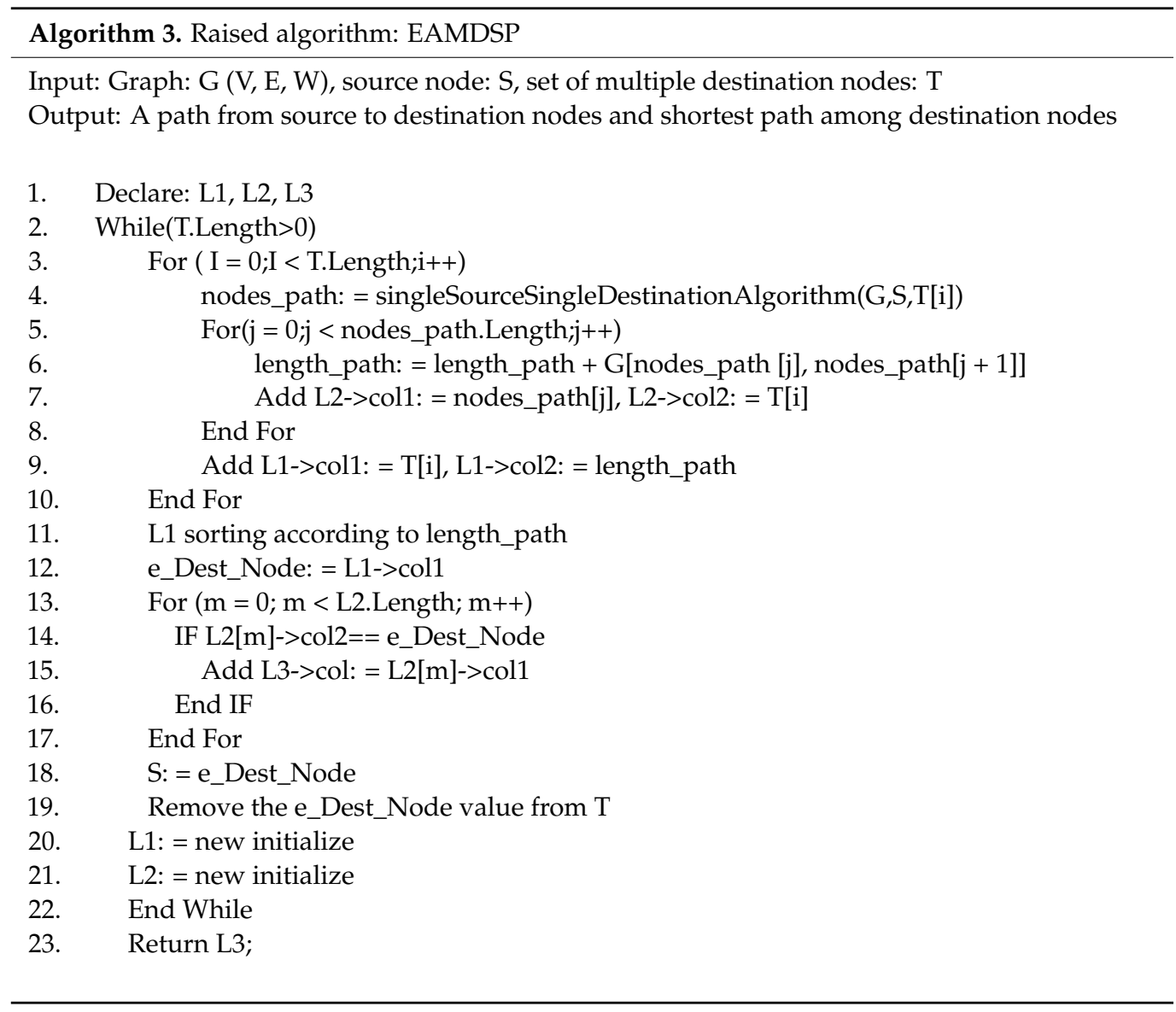

In the above algorithm, the variables L1 and L2 are declared as a two-dimensional array and L3 is a one-dimensional array for data storage. The first "while" loop runs until the last destination nodes come. The first "for" loop runs until the number of destination nodes are exhausted. The single-source single-destination algorithm is called to find the shortest path for visiting nodes among the source nodes to each destination node. The second "for" loop runs until the total number of shortest paths finding is completed for visiting nodes. Then the total path length or weight is calculated among the shortest path of visited nodes. Store each visited node into col1 of L2 and each destination node into col2 of L2. After that, store each destination node into col1 of L1 and path length or weight from a source to each destination node into col2 of L1. Sorting L1 according to path length or weight (smallest to biggest). Top each destination node from L1 $\rightarrow$ col1 first index (the smallest distance). Another new "for" loop is started for looping until L2 length or several destination nodes are covered. IF condition L2 [m] $\rightarrow$ col2 $==$ e_Dest_Node, then store the visiting nodes from a source to a destination in L3. S: = e_Dest_Node, replace current destination node value to source node value like the previous destination node is the next source node. Remove each destination node value from D. Then, L1: = new initialize or 
empty and L2: = new initialize or empty. Finally return L3, where L3 contains the source to all destination visiting nodes with the shortest path.

In Figure $5, p h_{u}$ is the shortest path and $p^{\prime} h_{u}$ is an alternative path $\mathrm{s} \rightarrow u$ via hubs $\mathrm{i}$ and $\mathrm{j}$. $\mathrm{S}$ is a set (shady zone) of all hubs whose ultimate smallest pathway from the source $\mathrm{s}$ has been ascertained.

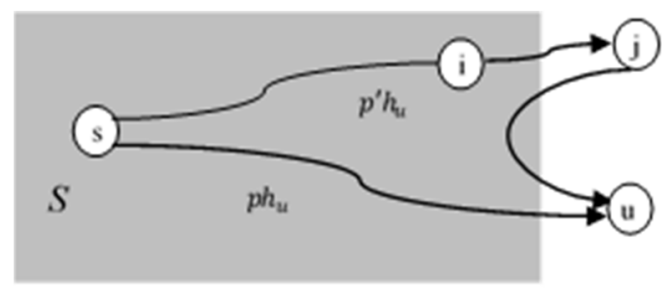

Figure 5. Source and destination shortest pathfinding in graphical representation.

Theorem 1. (Exactness) The ultimate smallest path among destination nodes $d_{i} \in D$ is constantly provided by EAMDSP, for example, $d_{i} . t=\delta$ path $\left(s, s d_{i}\right), i \in I$. The penultimate set $T_{i}$ contains several nodes, which are linked to the destination node $d_{i}$.

Proof. For each $u \in V$ and $t \in T$ assuming the algorithm found the label $u$.t, as well as the smallest path's weight, is $\delta$ path $(s, u)$ for $s \rightarrow u$. It aims to prove $u . t=\delta$ path $(s, u)$ where each vertex $u$ returned at the end of the EAMDSP.

Initial case, $s . t=\delta$ path $(\mathrm{s}, \mathrm{s})=0$, when $S=\{\mathrm{s}\}$ for every $v \in S$ which considers $v$.t shows the real smallest path's weight among $s$ and $v$,

$$
v . t=\delta \text { path }(s, v)
$$

In this algorithm, -1 is used for $u$ as a subsequent vertex to set $S$ via path $p h_{u}$, therefore, $u . t=\delta p h_{u}$. It will prove this path to be the minimum distance,

$$
u . t=\delta \operatorname{path}(s, u)
$$

Assume the minimum distance $\mathrm{s} \rightarrow u$ is the alternate path $p^{\prime} h_{u}$ instead of the path $p h_{u}$. The cost of the path $p h_{u}$ is more than the cost of the path $p^{\prime} h_{u}$

$$
p h_{u}>p^{\prime} h_{u}=u . t
$$

Equation (4) is explained in Figure 2, the path $p^{\prime} h_{u}$, begins in $S$ as well as at a few points omits $S$ to arrive at the node $u$ that is as of now not in $S$. Assume $(m, n)$ is the 1st edge toward $p^{\prime} h_{u}$ that departs from S. Evidently, $w_{\text {path }(s, m)}+w_{\text {path }(m, n)}<\delta_{p^{\prime} h_{u}}$. While $\mathrm{m} \in \mathrm{S}$, m.t is the cost of the minimum distance $s \rightarrow m$ path by the inductive hypothesis, $m . t=\delta_{\text {path }(s, m)}$ $\leq w_{\text {path }(s, m)}$, which implies $m \cdot t+w_{(m, n)} \leq \delta_{p^{\prime} h_{u}}$. The algorithm must update n.t while $n$ is adjacent to $\mathrm{m}$, which means $n . t \leq m . t+w_{(m, n)}$. The $u$ must have the shortest value due to the algorithm picked $u$, in other words, $u$.t $\leq n$.t. To sum up these disparities, we present the outcomes in Section 4.

\section{Results and Discussion}

In this section, the detailed simulation results of EAMDSP, CDSSSD, and MDMSMD will be presented. To evaluate the proposed EAMDSP simulation performance CDSSSD and MDMSMD simulation results on the same layout were used. The performance was evaluated concerning the number of nodes visited and the weight or cost. Two different scenarios, one was the car spare parts shop and another was the supermarket used for getting the comprehensive results. In the car spare parts shop scenario, the simulations were run in six unique phases as in four, six, eight, ten, twelve, and fifteen items searching layout. Three shortest path algorithms named CDSSSD, MDMSMD, and EAMDSP were used on 
both scenarios in different phases for getting a different result. The results comparisons are showed in the layout simulation, the summary table, and the graph.

Figure 6 shows the layout of a spare parts shop containing shelves (nodes) and road networks. The road network also consists of nodes. Every node has a unique number with longitude and latitude. The road network's one node is connected to the neighbor node with edge and weight value. The product/item is placed on the shelve node. The shelf node is connected to the road network's node with an edge and the edge has a weight value. When searching for a product, the product's shelf node number is considered to be the destination node. The shortest path was calculated between the source point (starting point) and the destination node, using the minimum the total nodes visited and their minimum weight or cost.

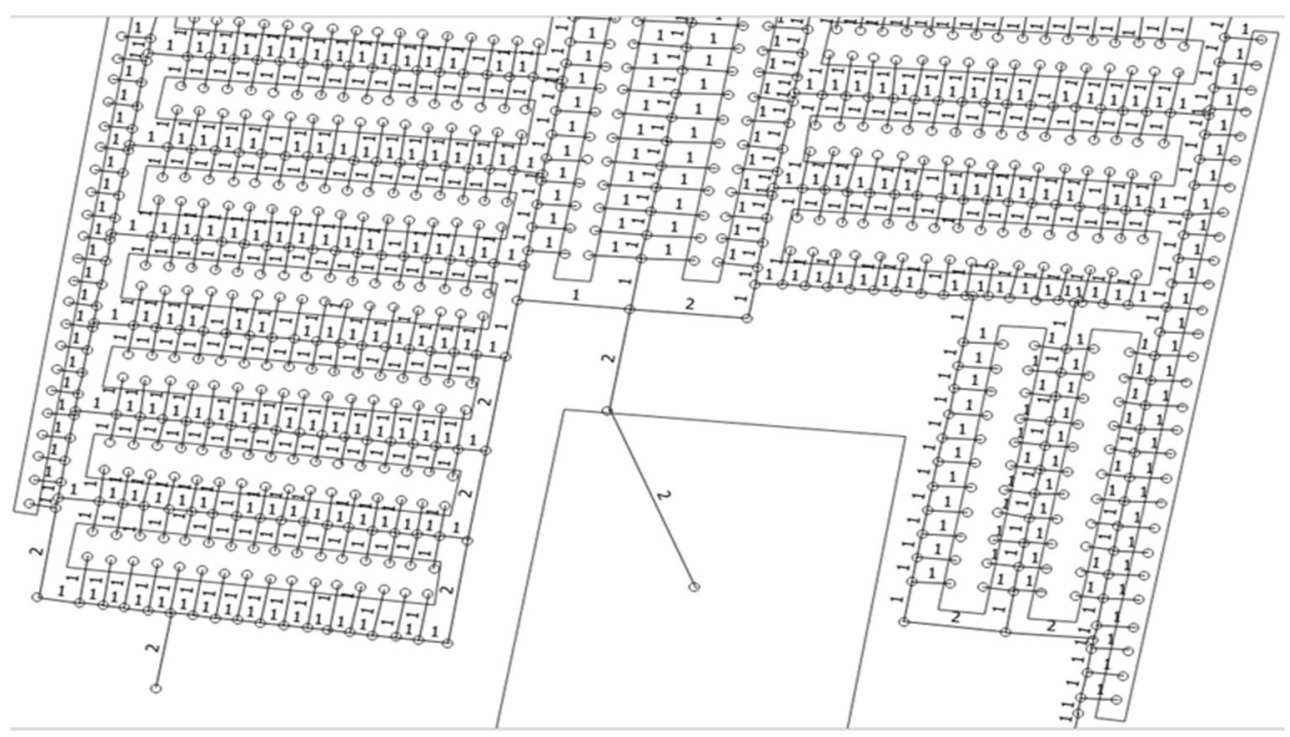

Figure 6. Drawing layout in a car spare part shop with nodes.

Table 1 represents the input which is the randomly selected four items. These input items were used for the same simulation layout of three algorithms such as CDSSSD, MDMSMD, and proposed EAMDSP.

Table 1. Selected four items and categories (input).

\begin{tabular}{cc}
\hline Item & Category \\
\hline Antenna cable & Audio/video devices \\
Bumper & Body components, including trim \\
Adjusting mechanism & Braking system \\
Bucket seat & Car seat \\
\hline
\end{tabular}

Figure 7a-c are car spare parts shop simulation result of EAMDSP, CDSSSD and MDMSMD respectively, which show the shortest path among four items. Table 2 shows the output summary data for the four-item search. EAMDSP for the shortest path between the above four items visited 93 nodes and had a total weight of 95. However, using CDSSSD and MDMSMD for the same items, the visited nodes were 124 and 120 and the total cost was 136 and 125, respectively. Therefore, the proposed EAMDSP is efficient for visited nodes and total weight. Finally, total visited nodes or total cost can be expressed as EAMDSP $<$ MDMSMD $<$ CDSSSD. The detailed data for the four items are shown in Appendix A. 


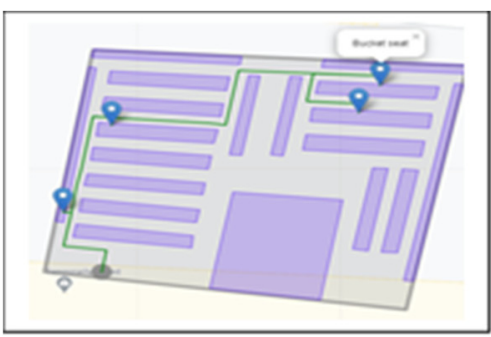

(a) EAMDSP

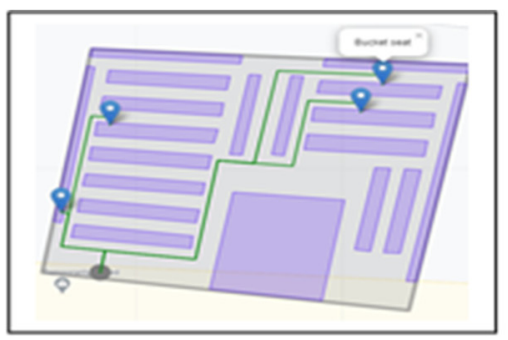

(b) CDSSSD

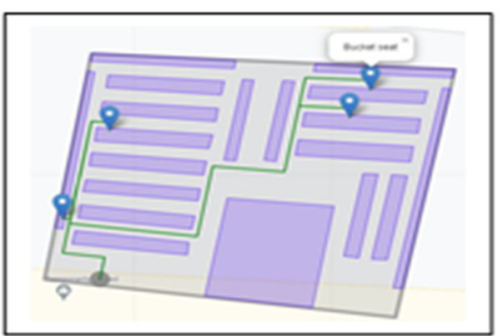

(c) MDMSMD

Figure 7. Simulation on a car spare parts shop scenario using EAMDSP, CDSSSD, and MDMSMD for a four-item search (output scenario).

Table 2. Summary data of EAMDSP, CDSSSD, and MDMSMD for a four-item search.

\begin{tabular}{cccc}
\hline SL No. & Algorithm Name & Total Visited Nodes & Total Cost \\
\hline 1 & EAMDSP & 93 & 95 \\
2 & CDSSSD & 124 & 136 \\
3 & MDMSMD & 120 & 125 \\
\hline
\end{tabular}

Table 3 represents the input value, which is six items randomly selected. These input items were used on the same simulation layout as the three algorithms CDSSSD, MDMSMD, and EAMDSP.

Table 3. (Input) Selecting six items and category.

\begin{tabular}{cc}
\hline Item & Category \\
\hline Antenna assembly & Audio/video devices \\
ABS steel pin & Braking system \\
Back seat & Car seat \\
Door switch & Electrical switches \\
Distributor cap & Engine components and parts \\
Oil gasket & Engine oil systems \\
\hline
\end{tabular}

Figure $8 \mathrm{a}-\mathrm{c}$ are car spare parts shop simulation result of EAMDSP, CDSSSD and MDMSMD, respectively which show the shortest path among six items. Table 4 shows the output summary data for the six-item search. EAMDSP for the shortest path between the six items visited 129 nodes and the total weight was 131. However, when using CDSSSD and MDMSMD for the same items, the visited nodes were 220 and 175 and the total cost was 242 and 179, respectively. Therefore, EAMDSP is efficient in terms of visited nodes and total weight. Finally, total visited nodes, or total cost can be expressed as EAMDSP $<$ MDMSMD $<$ CDSSSD.

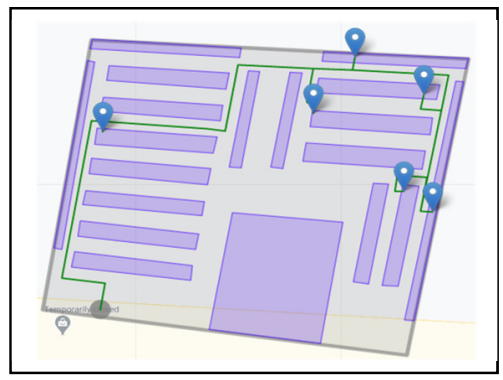

(a) EAMDSP

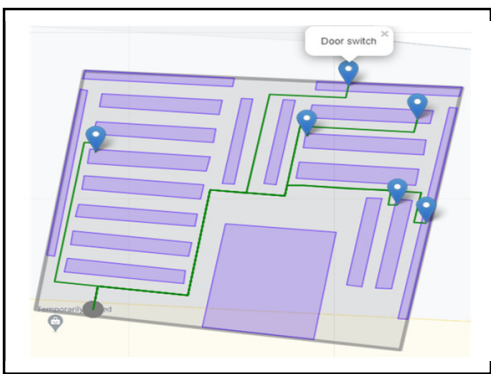

(b) CDSSSD

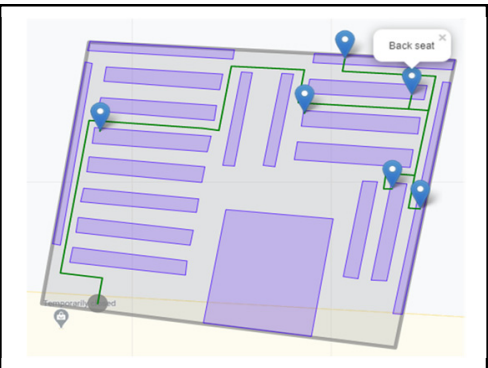

(c) MDMSMD

Figure 8. Simulation on a car spare parts shop scenario using EAMDSP, CDSSSD, and MDMSMD for a six-item search (output scenario). 
Table 4. Summary data of EAMDSP, CDSSSD, and MDMSMD for a six-item search.

\begin{tabular}{cccc}
\hline SL No. & Algorithm Name & Total Visited Nodes & Total Cost \\
\hline 1 & EAMDSP & 129 & 131 \\
2 & CDSSSD & 220 & 242 \\
3 & MDMSMD & 175 & 179 \\
\hline
\end{tabular}

Table 5 represents the input values of the eight items. These input items were used for the same simulation layout using three algorithms CDSSSD, MDMSMD, and EAMDSP.

Table 5. (Input) Selecting eight items and category.

\begin{tabular}{cc}
\hline Item & Category \\
\hline Radio and media player & Audio/video devices \\
Cowl screen & Body components, including trim \\
Adjusting mechanism (adjuster star wheel) & Braking system \\
Dashcam & Cameras \\
Bench seat & Car seat \\
Central locking & Doors \\
Fan ditch & Electrical switches \\
Engine compartment harness & Wiring harnesses \\
\hline
\end{tabular}

Figure $9 \mathrm{a}-\mathrm{c}$ are car spare parts shop simulation result of respectively EAMDSP, CDSSSD and MDMSMD which show the shortest path among eight items. Table 6 shows the output summary data for the eight items searched. When using EAMDSP for the shortest path between the above eight items, 119 nodes were visited, and the total weight or cost was 121. However, when using CDSSSD and MDMSMD for the same items, the visited nodes were 271 and 227 and the total cost was 297 and 239, respectively. So, EAMDSP is efficient for visited nodes and the total weight or cost. Finally, total visited nodes, or total cost can be expressed as EAMDSP $<$ MDMSMD $<$ CDSSSD.

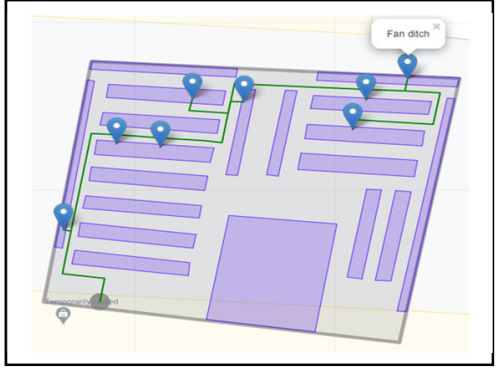

(a) EAMDSP

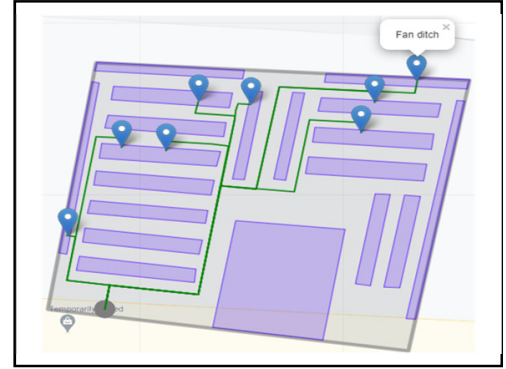

(b) CDSSSD

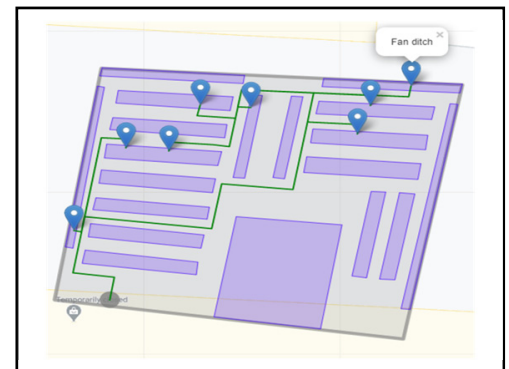

(c) MDMSMD

Figure 9. Simulation on a car spare parts shop scenario using EAMDSP, CDSSSD, and MDMSMD for an eight-item search (output scenario).

Table 6. Summary data of EAMDSP, CDSSSD, and MDMSMD for an eight-item search.

\begin{tabular}{cccc}
\hline SL No. & Algorithm Name & Total Visited Nodes & Total Cost \\
\hline 1 & EAMDSP & 119 & 121 \\
2 & CDSSSD & 271 & 297 \\
3 & MDMSMD & 227 & 239 \\
\hline
\end{tabular}

Table 7 represents the input value of ten items. These input items were used for the same simulation layout of three algorithms CDSSSD, MDMSMD, and EAMDSP. 
Table 7. (Input) Selecting ten items and category.

\begin{tabular}{cc}
\hline Item & Category \\
\hline Radiator core support & Body components \\
Brake pad & Braking system \\
Fan ditch & Electrical switches \\
Camshaft follower & Engine components and parts \\
PCV valve & Engine components and parts \\
Oil suction filter & Engine oil systems \\
Battery box & Low voltage electrical supply system \\
Alarm and siren & Miscellaneous \\
Wiring connector & Miscellaneous \\
Glowplug & Starting system \\
\hline
\end{tabular}

Figure 10a-c are car spare parts shop simulation result of EAMDSP, CDSSSD and MDMSMD respectively, which show the shortest path among ten items. Table 8 shows the output summary data for the ten-item search. When using EAMDSP for the shortest path between the above 10 items, 164 nodes were visited and the total weight or cost was 170. However, when using CDSSSD and MDMSMD for the same items, the visited nodes were 377 and 227, and the total cost was 414 and 236, respectively. Therefore, EAMDSP is efficient for visited nodes and total weight or cost. Finally, total visited nodes, or total cost can be expressed as EAMDSP $<$ MDMSMD $<$ CDSSSD.

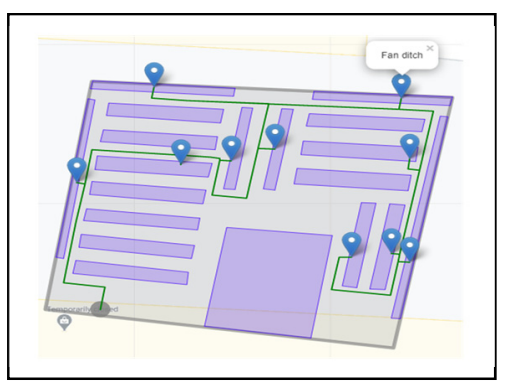

(a) EAMDSP

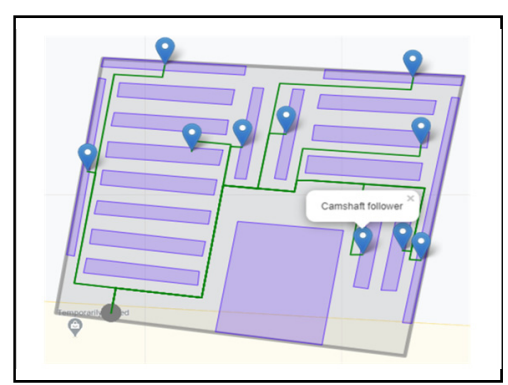

(b) CDSSSD

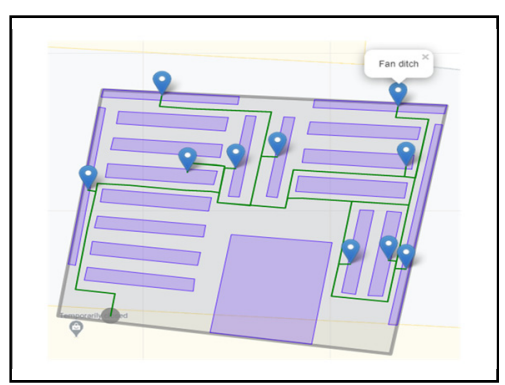

(c) MDMSMD

Figure 10. Simulation on a car spare parts shop scenario using EAMDSP, CDSSSD, and MDMSMD for a ten-item search (output scenario).

Table 8. Summary data of EAMDSP, CDSSSD, and MDMSMD for a ten-item search.

\begin{tabular}{cccc}
\hline SL No. & Algorithm Name & Total Visited Nodes & Total Cost \\
\hline 1 & EAMDSP & 164 & 170 \\
2 & CDSSSD & 377 & 414 \\
3 & MDMSMD & 227 & 236 \\
\hline
\end{tabular}

Table 9 represents the input value of twelve items. These input items were used for the same simulation layout of three algorithms CDSSSD, MDMSMD, and EAMDSP.

Figure 11a-c are car spare parts shop simulation result of EAMDSP, CDSSSD and MDMSMD respectively, which show the shortest path among twelve items. Table 10 shows the output summary data for the twelve items searched. When using EAMDSP for the shortest path between the above twelve items, 213 nodes were visited and the total weight or cost was 220. However, when using CDSSSD and MDMSMD for the same items, the visited nodes were 378 and 315 and the total cost was 408 and 327, respectively. Therefore, EAMDSP is efficient for visited nodes and total weight or cost. Finally, total visited nodes, or total cost can be expressed as EAMDSP $<$ MDMSMD $<$ CDSSSD. 
Table 9. (Input) Selecting twelve items and category.

\begin{tabular}{cc}
\hline Item & Category \\
\hline Tuner & Audio/video devices \\
Fascia rear and support & Body components \\
Brake backing plate & Braking system \\
Front seat & Car seat \\
Front Right Outer door handle & Doors \\
Ignition switch & Electrical switches \\
Fuel gauge & Gauges and meters \\
Ignition magneto & Ignition system \\
Side lighting & Lighting and signaling system \\
Coolant temperature sensor & Sensors \\
Sunroof glass & Windows \\
Air conditioning harness & Wiring harnesses \\
\hline
\end{tabular}

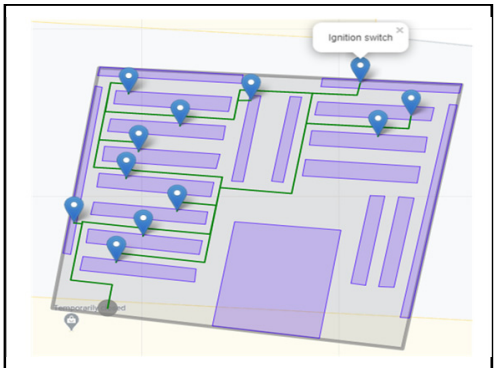

(a) EAMDSP

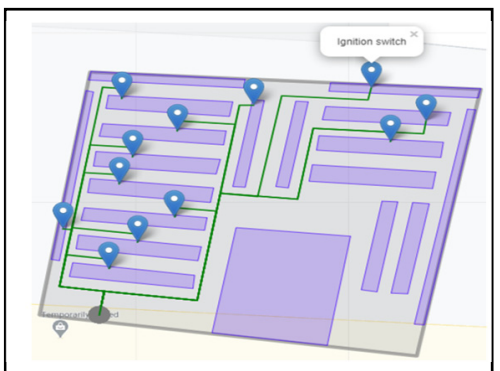

(b) CDSSSD

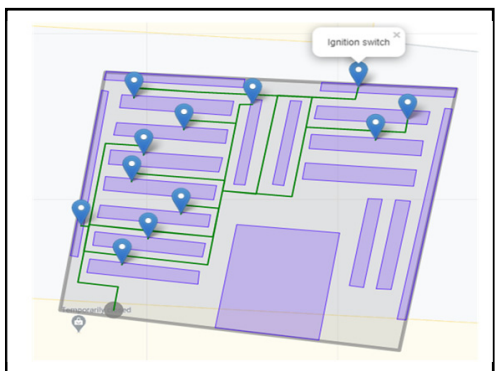

(c) MDMSMD

Figure 11. Simulation on a car spare parts shop scenario using EAMDSP, CDSSSD, and MDMSMD for a twelve-item search (output scenario).

Table 10. Summary data of EAMDSP, CDSSSD, and MDMSMD for a twelve-item search.

\begin{tabular}{cccc}
\hline SL No. & Algorithm Name & Total Visited Nodes & Total Cost \\
\hline 1 & EAMDSP & 213 & 220 \\
2 & CDSSSD & 378 & 408 \\
3 & MDMSMD & 315 & 327 \\
\hline
\end{tabular}

Table 11 represents the input values of the selected fifteen items. These input items were used for the same simulation layout of three algorithms CDSSSD, MDMSMD, and EAMDSP.

Table 11. (Input) Selecting fifteen items and categories.

\begin{tabular}{cc}
\hline Item & Category \\
\hline Front fascia and header panel & Body components \\
Brake disc & Braking system \\
Brake booster hose & Braking system \\
Backup camera & Cameras \\
Headrest & Car seat \\
Rear left side outer door handle & Doors \\
Switch cover & Electrical switches \\
Odometer & Gauges and meters \\
Distributor & Ignition system \\
Engine bay lighting & Lighting and signaling system \\
Shift improver & Miscellaneous \\
Knock sensor & Sensors \\
Starter drive & Starting system \\
Front left side door glass & Windows \\
Floor harness & Wiring harnesses \\
\hline
\end{tabular}


Figure 12a-c are car spare parts shop simulation result of EAMDSP, CDSSSD and MDMSMD respectively, which show the shortest path among fifteen items. Table 12 shows the output summary data for the fifteen-item search. When using EAMDSP for the shortest path among the above fifteen items, 225 nodes were visited and the total weight or cost was 233. However, when using CDSSSD and MDMSMD for the same items, the visited nodes were 487 and 393 and the total cost was 525 and 409, respectively. Therefore, our proposed shortest path algorithm EAMDSP is efficient for visited nodes and total weight or cost. Finally, total visited nodes, or total cost can be expressed as EAMDSP $<$ MDMSMD $<$ CDSSSD.

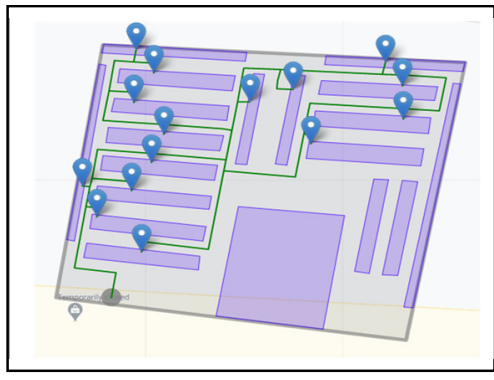

(a) EAMDSP

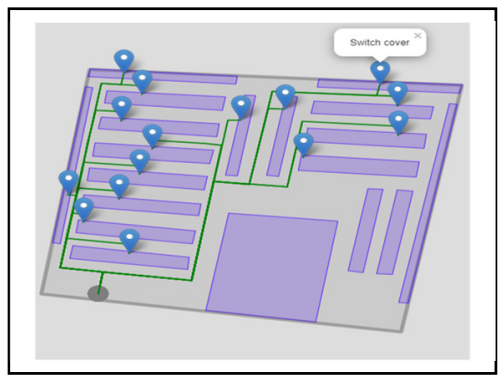

(b) CDSSSD

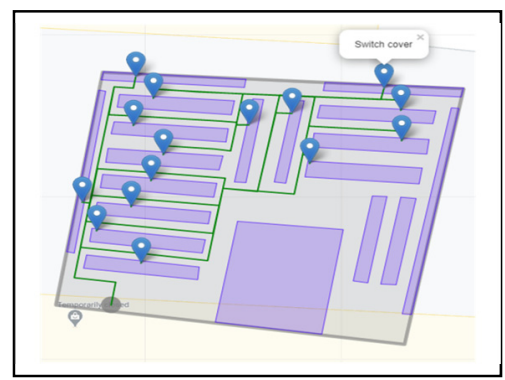

(c) MDMSMD

Figure 12. Simulation on a car spare parts shop scenario using EAMDSP, CDSSSD, and MDMSMD for a fifteen-item search (output scenario).

Table 12. Summary data of EAMDSP, CDSSSD, and MDMSMD for a fifteen-item search.

\begin{tabular}{cccc}
\hline SL No. & Algorithm Name & Total Visited Nodes & Total Cost \\
\hline 1 & EAMDSP & 225 & 233 \\
2 & CDSSSD & 487 & 525 \\
3 & MDMSMD & 393 & 409 \\
\hline
\end{tabular}

Figure 13 shows the cost-wise comparison among EAMDSP, CDSSSD and MDMSMD as per Table 13.

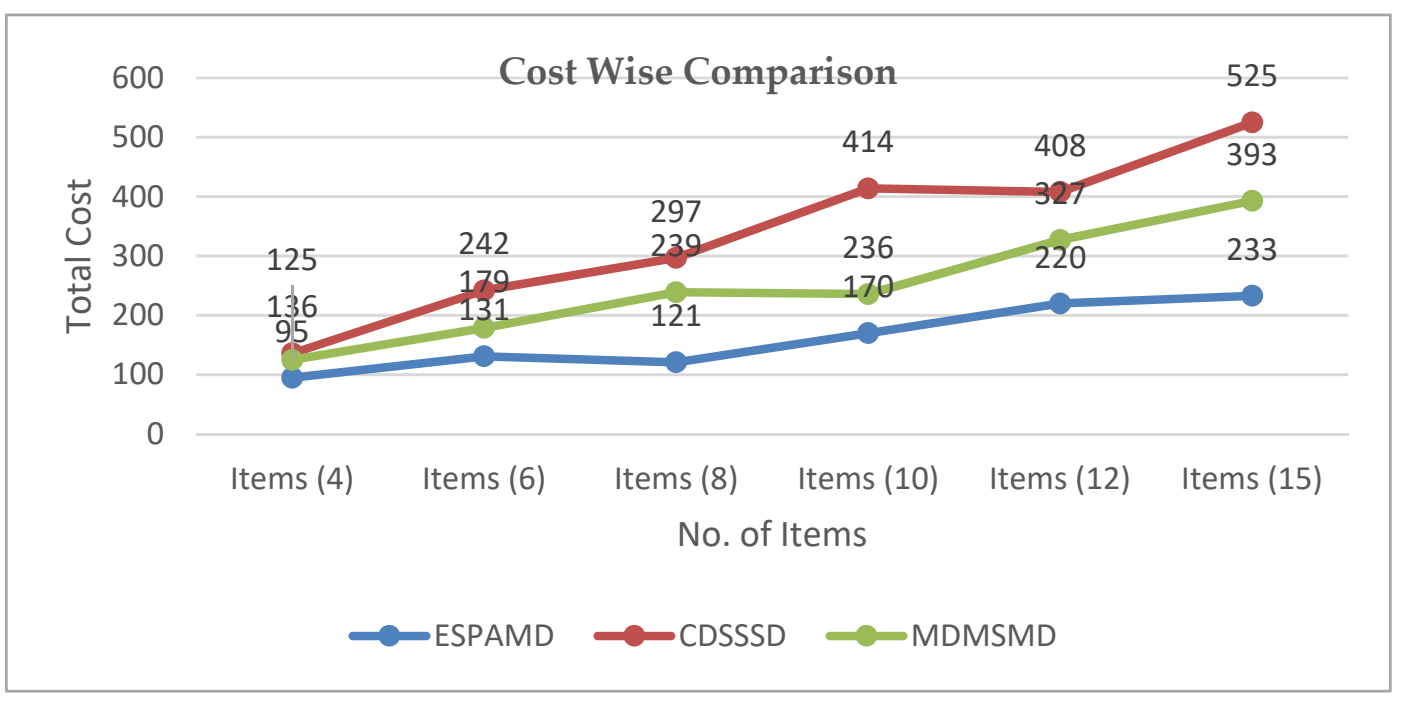

Figure 13. Cost-wise comparison among EAMDSP, CDSSSD, and MDMSMD. 
Table 13. Total cost-wise comparison among EAMDSP, CDSSSD, and MDMSMD.

\begin{tabular}{cccccccc}
\hline SL No. & $\begin{array}{c}\text { Algorithm } \\
\text { Name }\end{array}$ & Items (4) & Items (6) & Items (8) & $\begin{array}{c}\text { Items } \\
\mathbf{( 1 0 )}\end{array}$ & $\begin{array}{c}\text { Items } \\
\mathbf{( 1 2 )}\end{array}$ & $\begin{array}{c}\text { Items } \\
\text { (15) }\end{array}$ \\
\hline 1 & EAMDSP & 95 & 131 & 121 & 170 & 220 & 233 \\
2 & CDSSSD & 136 & 242 & 297 & 414 & 408 & 525 \\
3 & MDMSMD & 125 & 179 & 239 & 236 & 327 & 409 \\
\hline
\end{tabular}

Figure 14 shows the visited-wise comparison among EAMDSP, CDSSSD and MDMSMD as per Table 14 .

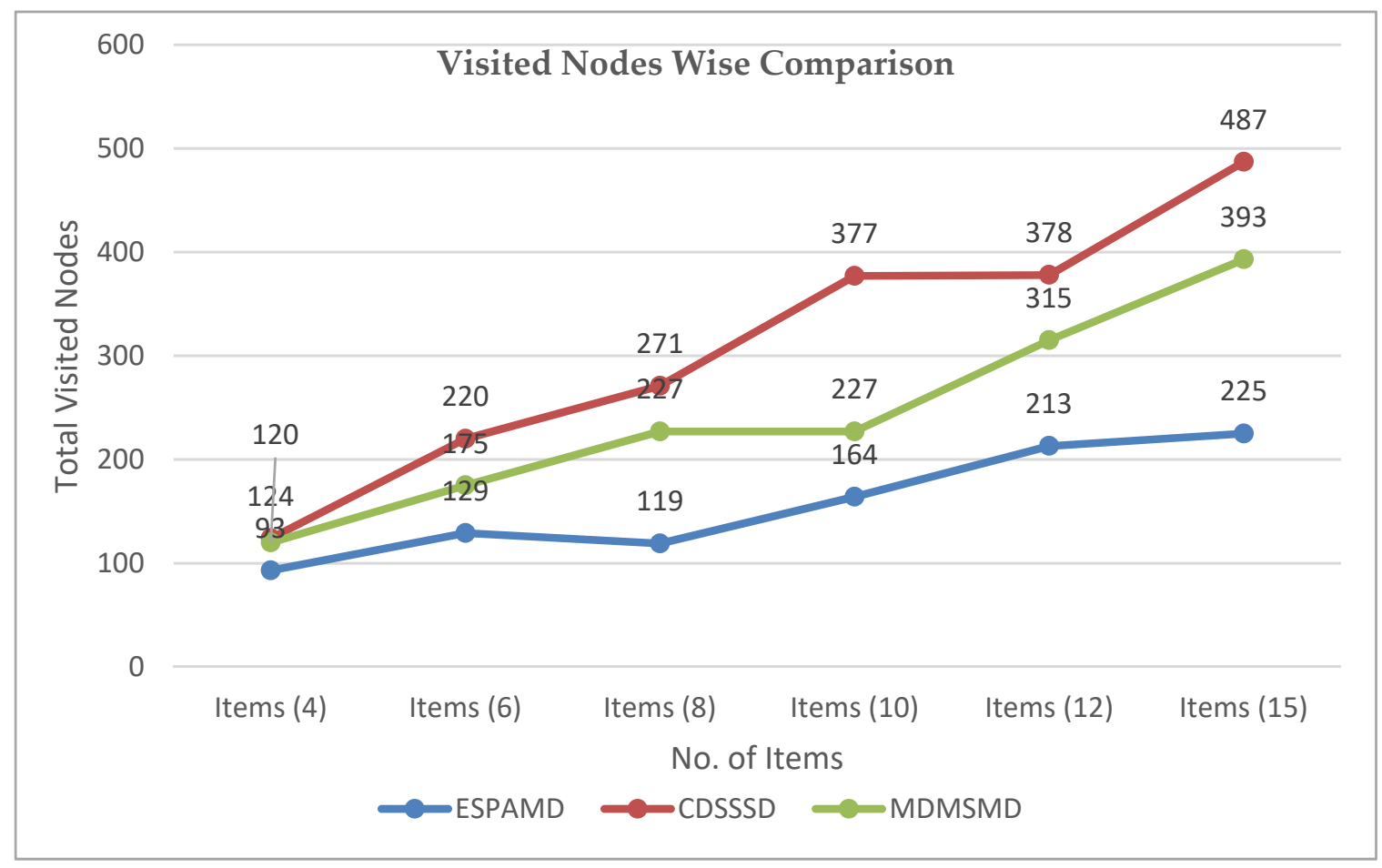

Figure 14. Visited nodes-wise comparison between EAMDSP, CDSSSD, and MDMSMD.

Table 14. Total visited node-wise comparison among EAMDSP, CDSSSD, and MDMSMD.

\begin{tabular}{cccccccc}
\hline SL No. & $\begin{array}{c}\text { Algorithm } \\
\text { Name }\end{array}$ & Items (4) & Items (6) & Items (8) & $\begin{array}{c}\text { Items } \\
\mathbf{( 1 0 )}\end{array}$ & $\begin{array}{c}\text { Items } \\
\mathbf{( 1 2 )}\end{array}$ & $\begin{array}{c}\text { Items } \\
\mathbf{( 1 5 )}\end{array}$ \\
\hline 1 & EAMDSP & 93 & 129 & 119 & 164 & 213 & 225 \\
2 & CDSSSD & 124 & 220 & 271 & 377 & 378 & 487 \\
3 & MDMSMD & 120 & 175 & 227 & 227 & 315 & 393 \\
\hline
\end{tabular}

In Figure 15, we present another scenario similar to a supermarket simulation scenario, the simulations were run in three unique phases as a five, eight, and eleven-item searching layout. Three shortest path algorithms CDSSSD, MDMSMD, and our proposed EAMDSP were used in this scenario's different phases for getting the different results and comparing results shown in the layout simulation. Summary data is presented in tables and graphs. 


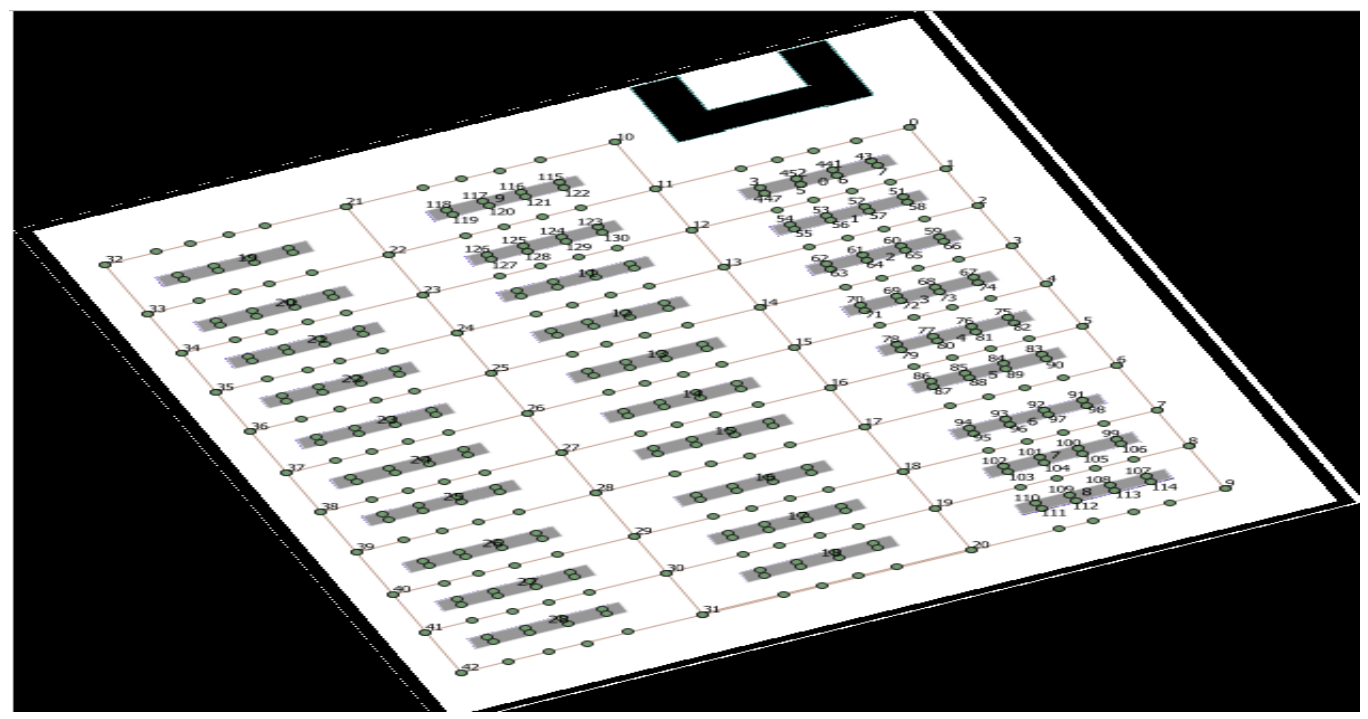

Figure 15. Drawing layout in the supermarket with nodes.

Table 15 represents the input value, which was randomly selected for five items. These input items were used for the same simulation layout of three algorithms CDSSSD, MDMSMD, and EAMDSP.

Table 15. (Input) Selecting five items and categories.

\begin{tabular}{cc}
\hline Item & Category \\
\hline Coffee & Beverages \\
Dinner rolls & Bread/Bakery \\
Vegetables & Canned/Jarred goods \\
Laundry detergent & Cleaners \\
Yogurt & Dairy \\
\hline
\end{tabular}

Figure $16 \mathrm{a}-\mathrm{c}$ are supermarket simulation result of respectively EAMDSP, CDSSSD and MDMSMD which show the shortest path among five items. Table 16 shows the output summary data for the five-item search. When using EAMDSP for the shortest path between the above the five items, 45 nodes were visited and the total weight was 54 . However, when using CDSSSD and MDMSMD for the same items, the visited nodes were 60 and 52 and the total cost was 84 and 69, respectively. Therefore, our proposed shortest path algorithm EAMDSP is efficient for visited nodes and total cost. Finally, total visited nodes, or total cost can be expressed as EAMDSP < MDMSMD < CDSSSD.

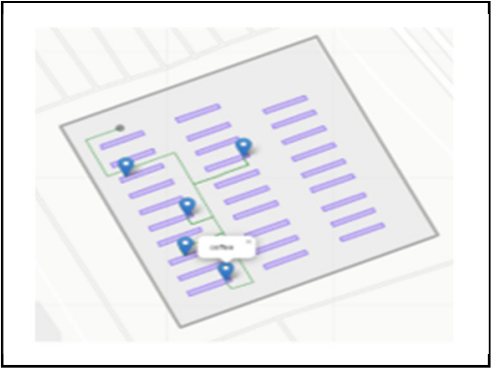

(a) EAMDSP

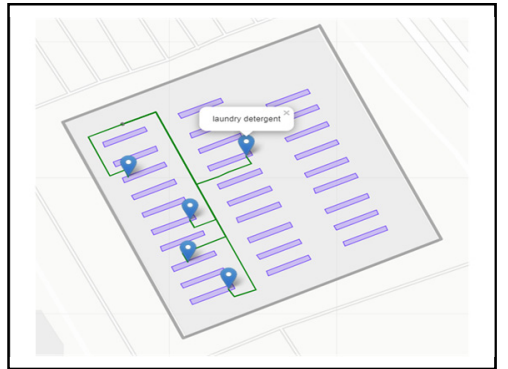

(b) CDSSSD

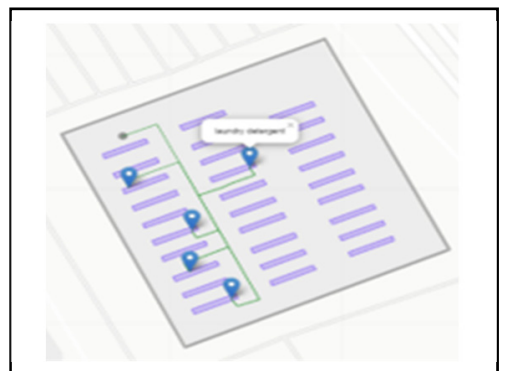

(c) MDMSMD

Figure 16. Simulation on supermarket scenario using EAMDSP, CDSSSD, and MDMSMD for a five-item search (output scenario). 
Table 16. The output summary data of EAMDSP, CDSSSD, and MDMSMD for a five-item search.

\begin{tabular}{cccc}
\hline SL No. & Algorithm Name & Total Visited Nodes & Total Cost \\
\hline 1 & EAMDSP & 45 & 54 \\
2 & CDSSSD & 60 & 84 \\
3 & MDMSMD & 52 & 69 \\
\hline
\end{tabular}

Table 17 represents the input values of the selected eight items. These input items were used for the same simulation layout of three algorithms CDSSSD, MDMSMD, and EAMDSP.

Table 17. (Input) Selecting eight items and categories.

\begin{tabular}{cc}
\hline Item & Category \\
\hline Soda & Beverages \\
Sandwich loaves & Bread/Bakery \\
Vegetables & Canned/Jarred goods \\
Dishwashing liquid & Cleaners \\
Eggs & Dairy \\
Mixes & Dry/Baking goods \\
Vegetables & Frozen foods \\
Pork & Meat \\
\hline
\end{tabular}

Figure $17 \mathrm{a}-\mathrm{c}$ are supermarket simulation result of respectively EAMDSP, CDSSSD and MDMSMD which show the shortest path among eight items. Table 18 shows the output summary data for the eight-item search. When using EAMDSP for the shortest path between the above eight items, 58 nodes were visited and the total weight was 67 . However, when using CDSSSD and MDMSMD for the same items, visited nodes were 104 and 72 and the total cost was 150 and 95, respectively. Therefore, EAMDSP is efficient for visited nodes and total weight. Finally, total visited nodes, or total cost can be expressed as EAMDSP $<$ MDMSMD $<$ CDSSSD.

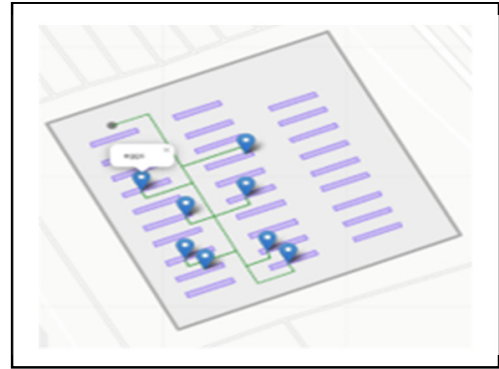

(a) EAMDSP

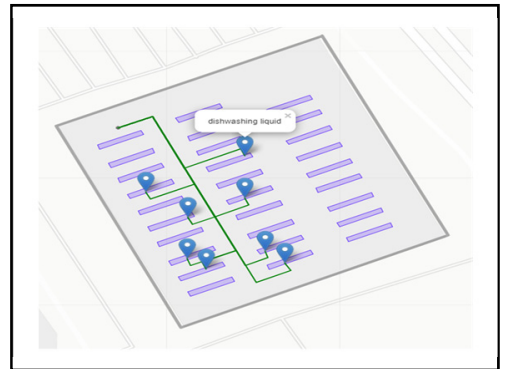

(b) CDSSSD

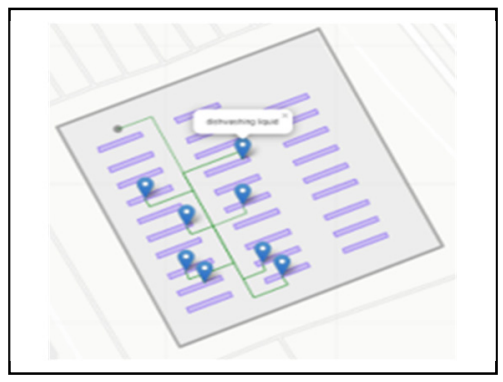

(c) MDMSMD

Figure 17. Simulation on supermarket scenario using EAMDSP, CDSSSD, and MDMSMD for an eight-item search (output scenario).

Table 18. The output summary data of EAMDSP, CDSSSD, and MDMSMD for an eight-item search.

\begin{tabular}{cccc}
\hline SL No. & Algorithm Name & Total Visited Nodes & Total Cost \\
\hline 1 & EAMDSP & 58 & 67 \\
2 & CDSSSD & 104 & 150 \\
3 & MDMSMD & 72 & 95 \\
\hline
\end{tabular}

Table 19 represents the input values of the selected eleven items. These input items were used for the same simulation layout of three algorithms CDSSSD, MDMSMD, and EAMDSP. 
Table 19. (Input) Selecting eleven items and categories.

\begin{tabular}{cc}
\hline Item & Category \\
\hline Juice & Beverages \\
Dinner rolls & Bread/Bakery \\
Spaghetti sauce & Canned/Jarred goods \\
Dishwashing detergent & Cleaners \\
Cheeses & Dairy \\
Flour & Dry/Baking goods \\
Individual meals & Frozen foods \\
Lunch meat & Meat \\
Batteries & Others \\
Paper towels & Paper goods \\
Shampoo & Personal cares \\
\hline
\end{tabular}

Figure 18a-c are supermarket simulation result of respectively EAMDSP, CDSSSD and MDMSMD which show the shortest path among eleven items. Table 20 shows the output summary data for an eleven-item search. When using EAMDSP for the shortest path between the above eleven items, 78 nodes were visited and the total weight was 88 . However, when using CDSSSD and MDMSMD for the same items the visited nodes were 124 and 101 and the total cost was 165 and 132 respectively. Therefore, EAMDSP is efficient for visited nodes and total weight. Finally, total visited nodes, or total cost can be expressed as EAMDSP $<$ MDMSMD < CDSSSD.

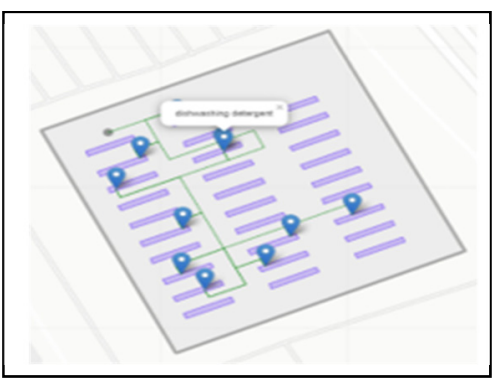

(a) EAMDSP

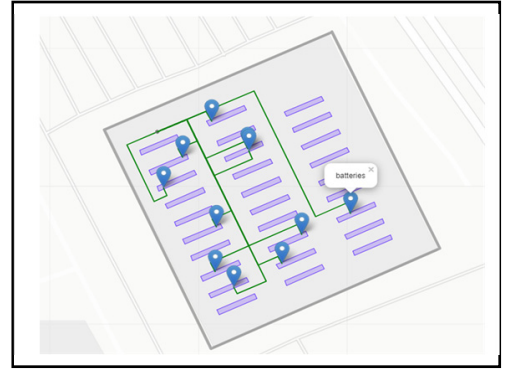

(b) CDSSSD

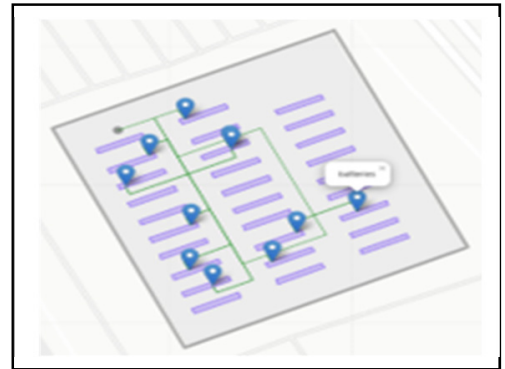

(c) MDMSMD

Figure 18. Simulation on supermarket scenario using EAMDSP, CDSSSD, and MDMSMD for an eleven-item search (output scenario).

Table 20. Summary data of EAMDSP, CDSSSD, and MDMSMD for eleven items search.

\begin{tabular}{cccc}
\hline SL No. & Algorithm Name & Total Nodes & Total Cost \\
\hline 1 & EAMDSP & 78 & 88 \\
2 & CDSSSD & 124 & 165 \\
3 & MDMSMD & 101 & 132 \\
\hline
\end{tabular}

Figure 19 shows the cost-wise comparison among EAMDSP, CDSSSD and MDMSMD as per Table 21.

Table 21. Total cost-wise comparison between EAMDSP, CDSSSD, and MDMSMD.

\begin{tabular}{ccccc}
\hline SL No. & Algorithm Name & Items (5) & Items (8) & Items (11) \\
\hline 1 & EAMDSP & 54 & 67 & 88 \\
2 & CDSSSD & 84 & 150 & 165 \\
3 & MDMSMD & 69 & 95 & 132 \\
\hline
\end{tabular}




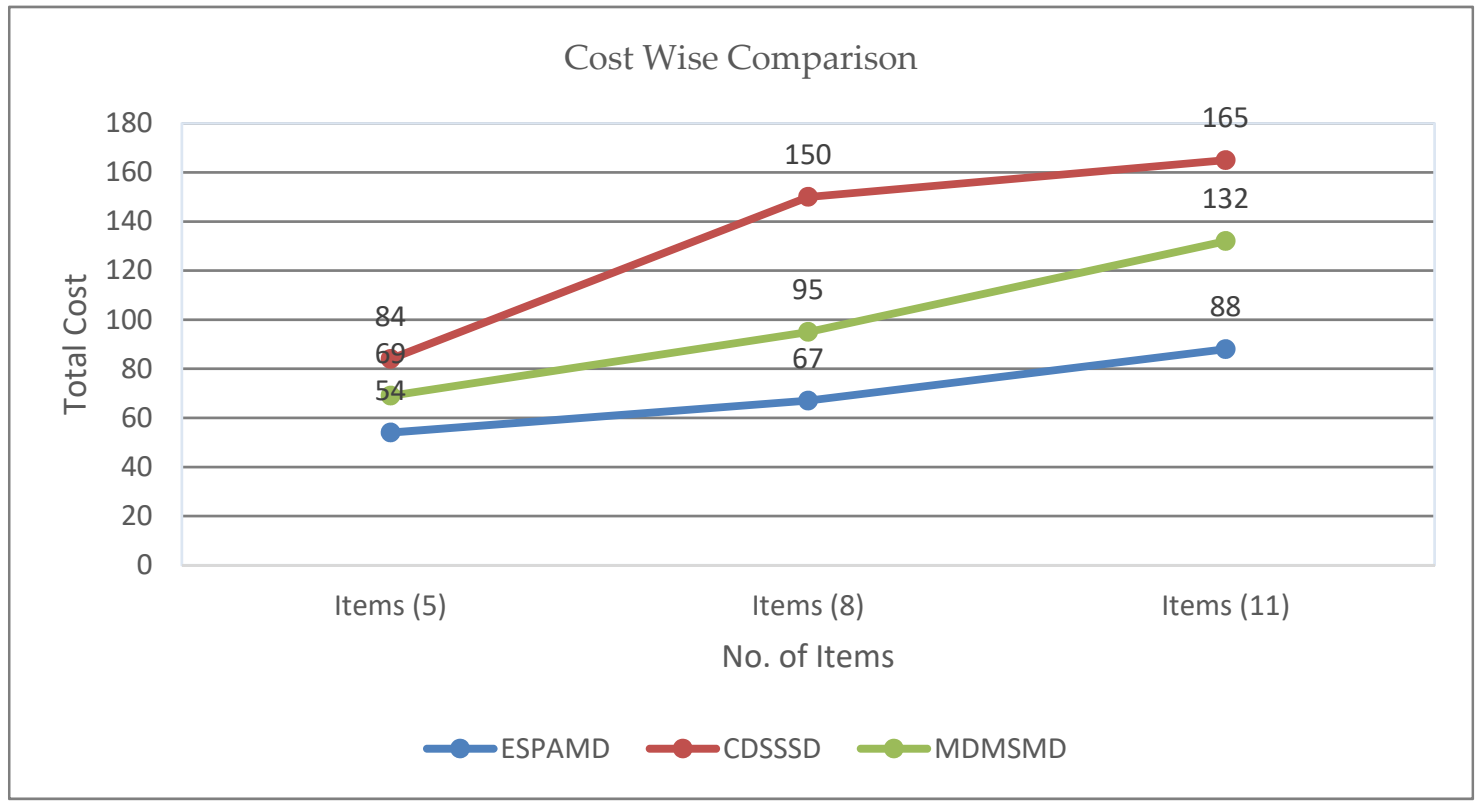

Figure 19. Cost-wise comparison between EAMDSP, CDSSSD, and MDMSMD.

Figure 20 shows the visited-wise comparison among EAMDSP, CDSSSD and MDMSMD as per Table 22 .

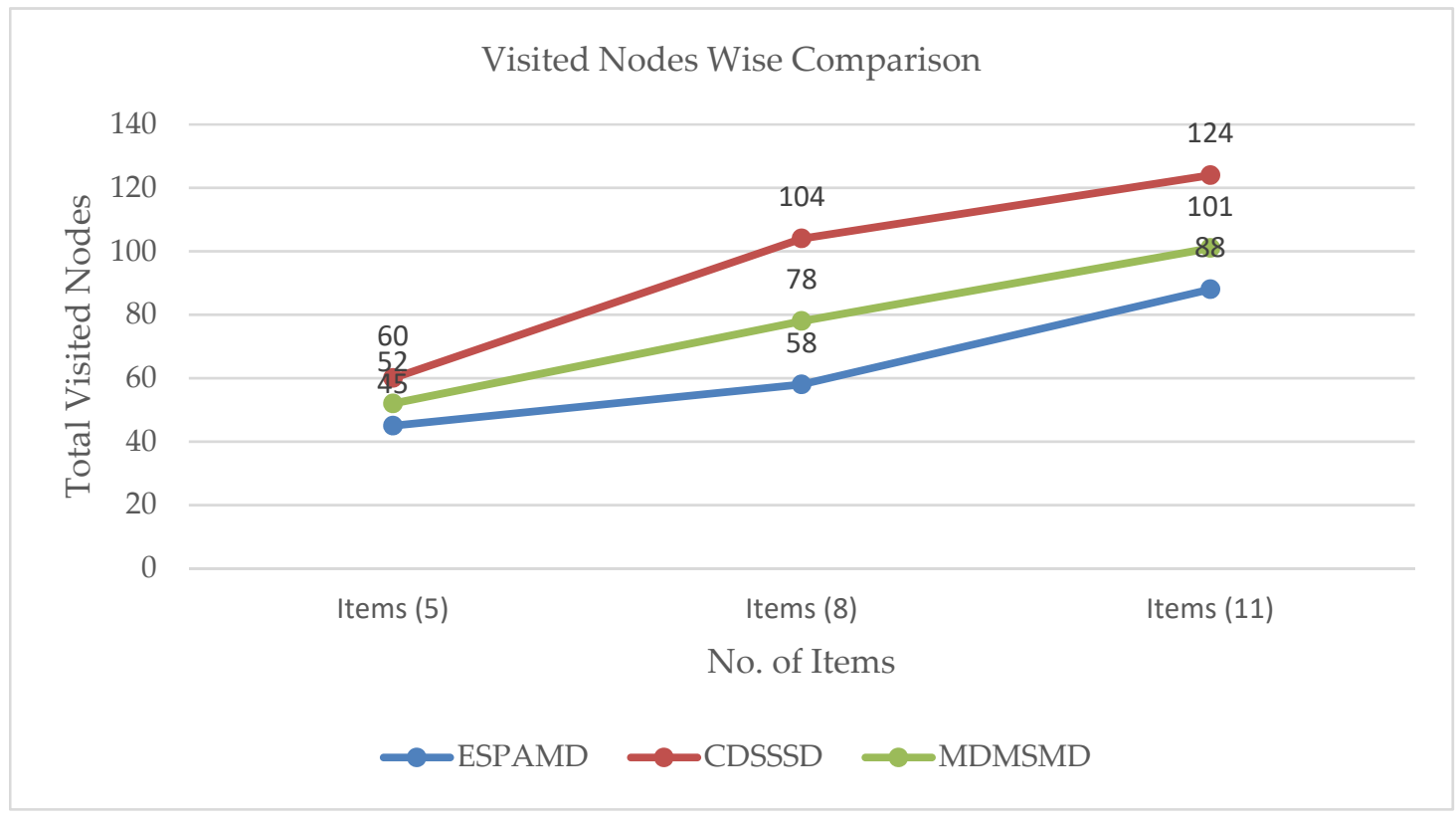

Figure 20. Visited node-wise comparison between EAMDSP, CDSSSD, and MDMSMD.

Table 22. Total visited node-wise comparison between EAMDSP, CDSSSD, and MDMSMD.

\begin{tabular}{ccccc}
\hline SL No. & Algorithm Name & Items (5) & Items (8) & Items (11) \\
\hline 1 & EAMDSP & 45 & 58 & 88 \\
2 & CDSSSD & 60 & 104 & 124 \\
3 & MDMSMD & 52 & 78 & 101 \\
\hline
\end{tabular}


Finally, based on Figures 13, 14, 19 and 20, and Tables 13, 14, 21 and 22 it is reported that the proposed EAMDSP demonstrates the outstanding performance on cost and number of visited nodes compared to CDSSSD and MDMSMD.

\section{Conclusions}

The proposed EAMDSP algorithm was developed and implemented in indoor applications and found to be more efficient for a multi-destination search. It can be used on any type of road network with multi-destinations for shortest path searching. In this paper, we evaluated the EAMDSP by comparing it with CDSSSD and MDMSMD using two different simulations of several items of searching. For a search with a large number of products, EAMDSP demonstrated an outstanding performance compared to CDSSSD and MDMSMD, based on the total visited nodes and weights. The output of these simulations based on visited nodes and the minimum sum of weights is presented in tables and graphs. This research can be a useful guide for indoor shortest-path researchers. In future work, we might extend the application of EAMDSP from an indoor to an outdoor environment.

Author Contributions: Conceptualization, M.A., F.H. and T.K.G.; methodology, M.A., F.H. and T.K.G.; validation, F.H. and T.K.G.; formal analysis, M.A. and F.H.; writing-original draft preparation, M.A. and F.H.; writing - review and editing, F.H., C.P.T. and S.S.; visualization, M.A.B. and S.A.; supervision, T.K.G. and S.S.; project administration, F.H.; funding acquisition, T.K.G., A.A. and H.-Y.W. All authors have read and agreed to the published version of the manuscript.

Funding: This research is also funded by the "Indoor Internet of Things (IOT) Tracking Algorithm Development based on Radio Signal Characterization" and "Mobile IOT: Location Aware" project bearing grant no. [FRGS/1/2018/TK08/MMU/02/1] and [MMUE/180025].

Institutional Review Board Statement: Not applicable.

Informed Consent Statement: Not applicable.

Data Availability Statement: Not applicable.

Acknowledgments: Special thanks to MMU RMC for providing comprehensive financial assistance to this research.

Conflicts of Interest: The authors declare no conflict of interest. The funding sponsors had no role in the design of the study, in the collection, analyses, or interpretation of data, in the writing of the manuscript, or in the decision to publish the results.

\section{Appendix A. Result Detail Data Tables}

Table A1. Simulation detail data for car spare parts scenario using EAMDSP for four items search.

\begin{tabular}{|c|c|c|c|c|c|c|c|c|}
\hline SL No. & $\begin{array}{l}\text { Visited } \\
\text { Node }\end{array}$ & Longitude & Latitude & $\begin{array}{c}\text { Source } \\
\text { Node }\end{array}$ & $\begin{array}{l}\text { Destination } \\
\text { Node }\end{array}$ & Weight & Item Name & \\
\hline 1 & 342 & 102.27257 & 2.2528471 & 342 & 384 & 0 & \multirow{15}{*}{ Bumper } & \multirow[t]{10}{*}{ 1st source } \\
\hline 2 & 208 & 102.27257 & 2.252859 & 342 & 384 & 2 & & \\
\hline 3 & 209 & 102.27257 & 2.2528595 & 342 & 384 & 1 & & \\
\hline 4 & 210 & 102.27257 & 2.2528599 & 342 & 384 & 1 & & \\
\hline 5 & 211 & 102.27256 & 2.2528603 & 342 & 384 & 1 & & \\
\hline 6 & 212 & 102.27256 & 2.2528607 & 342 & 384 & 1 & & \\
\hline 7 & 213 & 102.27256 & 2.2528615 & 342 & 384 & 1 & & \\
\hline 8 & 214 & 102.27256 & 2.2528757 & 342 & 384 & 2 & & \\
\hline 9 & 215 & 102.27256 & 2.2528774 & 342 & 384 & 1 & & \\
\hline 10 & 232 & 102.27256 & 2.2528798 & 342 & 384 & 1 & & \\
\hline 11 & 384 & 102.27256 & 2.2528801 & 342 & 384 & 1 & & \multirow[t]{5}{*}{$\begin{array}{c}1 \text { st } \\
\text { destination }\end{array}$} \\
\hline 12 & 232 & 102.27256 & 2.2528798 & 384 & 472 & 1 & & \\
\hline 13 & 233 & 102.27256 & 2.2528833 & 384 & 472 & 1 & & \\
\hline 14 & 234 & 102.27256 & 2.2528868 & 384 & 472 & 1 & & \\
\hline 15 & 235 & 102.27256 & 2.2528903 & 384 & 472 & 1 & & \\
\hline
\end{tabular}


Table A1. Cont.

\begin{tabular}{|c|c|c|c|c|c|c|c|c|}
\hline SL No. & $\begin{array}{l}\text { Visited } \\
\text { Node }\end{array}$ & Longitude & Latitude & $\begin{array}{l}\text { Source } \\
\text { Node }\end{array}$ & $\begin{array}{c}\text { Destination } \\
\text { Node }\end{array}$ & Weight & Item Name & \\
\hline 16 & 236 & 102.27256 & 2.2528911 & 384 & 472 & 1 & \multirow{55}{*}{$\begin{array}{l}\text { Antenna } \\
\text { cable }\end{array}$} & \multirow{55}{*}{$\begin{array}{c}\text { 2nd } \\
\text { destination }\end{array}$} \\
\hline 17 & 253 & 102.27256 & 2.2528938 & 384 & 472 & 1 & & \\
\hline 18 & 254 & 102.27256 & 2.2528971 & 384 & 472 & 1 & & \\
\hline 19 & 255 & 102.27256 & 2.2529008 & 384 & 472 & 1 & & \\
\hline 20 & 256 & 102.27256 & 2.2529042 & 384 & 472 & 1 & & \\
\hline 21 & 257 & 102.27256 & 2.2529051 & 384 & 472 & 1 & & \\
\hline 22 & 274 & 102.27256 & 2.2529077 & 384 & 472 & 1 & & \\
\hline 23 & 275 & 102.27256 & 2.2529111 & 384 & 472 & 1 & & \\
\hline 24 & 276 & 102.27256 & 2.2529148 & 384 & 472 & 1 & & \\
\hline 25 & 277 & 102.27257 & 2.2529182 & 384 & 472 & 1 & & \\
\hline 26 & 278 & 102.27257 & 2.2529192 & 384 & 472 & 1 & & \\
\hline 27 & 295 & 102.27257 & 2.2529217 & 384 & 472 & 1 & & \\
\hline 28 & 296 & 102.27257 & 2.2529252 & 384 & 472 & 1 & & \\
\hline 29 & 297 & 102.27257 & 2.2529287 & 384 & 472 & 1 & & \\
\hline 30 & 298 & 102.27257 & 2.2529323 & 384 & 472 & 1 & & \\
\hline 31 & 299 & 102.27257 & 2.2529332 & 384 & 472 & 1 & & \\
\hline 32 & 300 & 102.27257 & 2.2529328 & 384 & 472 & 1 & & \\
\hline 33 & 301 & 102.27258 & 2.2529326 & 384 & 472 & 1 & & \\
\hline 34 & 472 & 102.27258 & 2.252928 & 384 & 472 & 1 & & \\
\hline 35 & 301 & 102.27258 & 2.2529326 & 472 & 683 & 1 & & \\
\hline 36 & 302 & 102.27258 & 2.2529323 & 472 & 683 & 1 & & \\
\hline 37 & 303 & 102.27258 & 2.252932 & 472 & 683 & 1 & & \\
\hline 38 & 304 & 102.27259 & 2.2529318 & 472 & 683 & 1 & & \\
\hline 39 & 305 & 102.27259 & 2.2529315 & 472 & 683 & 1 & & \\
\hline 40 & 306 & 102.27259 & 2.2529314 & 472 & 683 & 1 & & \\
\hline 41 & 307 & 102.2726 & 2.2529311 & 472 & 683 & 1 & & \\
\hline 42 & 308 & 102.2726 & 2.2529308 & 472 & 683 & 1 & & \\
\hline 43 & 309 & 102.2726 & 2.2529306 & 472 & 683 & 1 & & \\
\hline 44 & 310 & 102.2726 & 2.2529303 & 472 & 683 & 1 & & \\
\hline 45 & 311 & 102.27261 & 2.2529301 & 472 & 683 & 1 & & \\
\hline 46 & 312 & 102.27261 & 2.2529298 & 472 & 683 & 1 & & \\
\hline 47 & 313 & 102.27261 & 2.2529295 & 472 & 683 & 1 & & \\
\hline 48 & 314 & 102.27262 & 2.2529293 & 472 & 683 & 1 & & \\
\hline 49 & 315 & 102.27262 & 2.2529291 & 472 & 683 & 1 & & \\
\hline 50 & 186 & 102.27262 & 2.2529285 & 472 & 683 & 1 & & \\
\hline 51 & 185 & 102.27262 & 2.252931 & 472 & 683 & 1 & & \\
\hline 52 & 184 & 102.27263 & 2.2529348 & 472 & 683 & 1 & & \\
\hline 53 & 183 & 102.27263 & 2.2529386 & 472 & 683 & 1 & & \\
\hline 54 & 182 & 102.27263 & 2.2529423 & 472 & 683 & 1 & & \\
\hline 55 & 181 & 102.27263 & 2.2529435 & 472 & 683 & 1 & & \\
\hline 56 & 180 & 102.27263 & 2.252946 & 472 & 683 & 1 & & \\
\hline 57 & 179 & 102.27263 & 2.2529495 & 472 & 683 & 1 & & \\
\hline 58 & 178 & 102.27263 & 2.2529534 & 472 & 683 & 1 & & \\
\hline 59 & 22 & 102.27263 & 2.2529585 & 472 & 683 & 1 & & \\
\hline 60 & 23 & 102.27265 & 2.2529578 & 472 & 683 & 1 & & \\
\hline 61 & 24 & 102.27266 & 2.2529569 & 472 & 683 & 2 & & \\
\hline 62 & 25 & 102.27266 & 2.2529567 & 472 & 683 & 1 & & \\
\hline 63 & 26 & 102.27267 & 2.2529567 & 472 & 683 & 1 & & \\
\hline 64 & 27 & 102.27267 & 2.2529564 & 472 & 683 & 1 & & \\
\hline 65 & 28 & 102.27267 & 2.2529563 & 472 & 683 & 1 & & \\
\hline 66 & 29 & 102.27268 & 2.2529562 & 472 & 683 & 1 & & \\
\hline 67 & 30 & 102.27268 & 2.2529559 & 472 & 683 & 1 & & \\
\hline 68 & 31 & 102.27268 & 2.2529558 & 472 & 683 & 1 & & \\
\hline 69 & 32 & 102.27269 & 2.2529557 & 472 & 683 & 1 & & \\
\hline 70 & 33 & 102.27269 & 2.2529554 & 472 & 683 & 1 & & \\
\hline 71 & 683 & 102.27269 & 2.2529501 & 472 & 683 & 1 & Bucket seat & $\begin{array}{c}\text { 3rd } \\
\text { destination }\end{array}$ \\
\hline
\end{tabular}


Table A1. Cont.

\begin{tabular}{|c|c|c|c|c|c|c|c|c|}
\hline SL No. & $\begin{array}{l}\text { Visited } \\
\text { Node }\end{array}$ & Longitude & Latitude & $\begin{array}{l}\text { Source } \\
\text { Node }\end{array}$ & $\begin{array}{l}\text { Destination } \\
\text { Node }\end{array}$ & Weight & Item Name & \\
\hline 72 & 33 & 102.27269 & 2.2529554 & 683 & 713 & 1 & & \\
\hline 73 & 32 & 102.27269 & 2.2529557 & 683 & 713 & 1 & & \\
\hline 74 & 31 & 102.27268 & 2.2529558 & 683 & 713 & 1 & & \\
\hline 75 & 30 & 102.27268 & 2.2529559 & 683 & 713 & 1 & & \\
\hline 76 & 29 & 102.27268 & 2.2529562 & 683 & 713 & 1 & & \\
\hline 77 & 28 & 102.27267 & 2.2529563 & 683 & 713 & 1 & & \\
\hline 78 & 27 & 102.27267 & 2.2529564 & 683 & 713 & 1 & & \\
\hline 79 & 26 & 102.27267 & 2.2529567 & 683 & 713 & 1 & & \\
\hline 80 & 25 & 102.27266 & 2.2529567 & 683 & 713 & 1 & & \\
\hline 81 & 24 & 102.27266 & 2.2529569 & 683 & 713 & 1 & & \\
\hline 82 & 164 & 102.27266 & 2.2529515 & 683 & 713 & 1 & & \\
\hline 83 & 163 & 102.27266 & 2.2529477 & 683 & 713 & 1 & & \\
\hline 84 & 162 & 102.27266 & 2.252944 & 683 & 713 & 1 & & \\
\hline 85 & 145 & 102.27266 & 2.2529414 & 683 & 713 & 1 & & \\
\hline 86 & 146 & 102.27266 & 2.2529411 & 683 & 713 & 1 & & \\
\hline 87 & 147 & 102.27267 & 2.2529409 & 683 & 713 & 1 & & \\
\hline 88 & 148 & 102.27267 & 2.2529407 & 683 & 713 & 1 & & \\
\hline 89 & 149 & 102.27267 & 2.2529405 & 683 & 713 & 1 & & \\
\hline 90 & 150 & 102.27267 & 2.2529404 & 683 & 713 & 1 & & \\
\hline 91 & 151 & 102.27268 & 2.2529402 & 683 & 713 & 1 & & \\
\hline 92 & 152 & 102.27268 & 2.25294 & 683 & 713 & 1 & & \\
\hline 93 & 713 & 102.27268 & 2.2529351 & 683 & 713 & 1 & $\begin{array}{l}\text { Adjusting } \\
\text { mechanism } \\
\text { (adjuster } \\
\text { star wheel) }\end{array}$ & $\begin{array}{c}\text { 4th } \\
\text { destination }\end{array}$ \\
\hline
\end{tabular}

Total visited nodes $=93$, total $\operatorname{cost}=95$.

Table A2. Simulation detail data for car spare parts Scenario using CDSSSD for four items search.

\begin{tabular}{|c|c|c|c|c|c|c|c|}
\hline SL No. & $\begin{array}{l}\text { Visited } \\
\text { Node }\end{array}$ & Longitude & Latitude & Source Node & $\begin{array}{l}\text { Destination } \\
\text { Node }\end{array}$ & Weight & Item Name \\
\hline 1 & 342 & 102.27257 & 2.2528471 & 342 & 472 & 0 & \\
\hline 2 & 208 & 102.27257 & 2.252859 & 342 & 472 & 2 & \\
\hline 3 & 209 & 102.27257 & 2.2528595 & 342 & 472 & 1 & \\
\hline 4 & 210 & 102.27257 & 2.2528599 & 342 & 472 & 1 & \\
\hline 5 & 211 & 102.27256 & 2.2528603 & 342 & 472 & 1 & \\
\hline 6 & 212 & 102.27256 & 2.2528607 & 342 & 472 & 1 & \\
\hline 7 & 213 & 102.27256 & 2.2528615 & 342 & 472 & 1 & \\
\hline 8 & 214 & 102.27256 & 2.2528757 & 342 & 472 & 2 & \\
\hline 9 & 215 & 102.27256 & 2.2528774 & 342 & 472 & 1 & \\
\hline 10 & 232 & 102.27256 & 2.2528798 & 342 & 472 & 1 & \\
\hline 11 & 233 & 102.27256 & 2.2528833 & 342 & 472 & 1 & \\
\hline 12 & 234 & 102.27256 & 2.2528868 & 342 & 472 & 1 & \\
\hline 13 & 235 & 102.27256 & 2.2528903 & 342 & 472 & 1 & \\
\hline 14 & 236 & 102.27256 & 2.2528911 & 342 & 472 & 1 & \\
\hline 15 & 253 & 102.27256 & 2.2528938 & 342 & 472 & 1 & \\
\hline 16 & 254 & 102.27256 & 2.2528971 & 342 & 472 & 1 & \\
\hline 17 & 255 & 102.27256 & 2.2529008 & 342 & 472 & 1 & \\
\hline 18 & 256 & 102.27256 & 2.2529042 & 342 & 472 & 1 & \\
\hline 19 & 257 & 102.27256 & 2.2529051 & 342 & 472 & 1 & \\
\hline 20 & 274 & 102.27256 & 2.2529077 & 342 & 472 & 1 & \\
\hline 21 & 275 & 102.27256 & 2.2529111 & 342 & 472 & 1 & \\
\hline 22 & 276 & 102.27256 & 2.2529148 & 342 & 472 & 1 & \\
\hline 23 & 277 & 102.27257 & 2.2529182 & 342 & 472 & 1 & \\
\hline 24 & 278 & 102.27257 & 2.2529192 & 342 & 472 & 1 & \\
\hline
\end{tabular}


Table A2. Cont.

\begin{tabular}{|c|c|c|c|c|c|c|c|}
\hline SL No. & $\begin{array}{l}\text { Visited } \\
\text { Node }\end{array}$ & Longitude & Latitude & Source Node & $\begin{array}{c}\text { Destination } \\
\text { Node }\end{array}$ & Weight & Item Name \\
\hline 25 & 295 & 102.27257 & 2.2529217 & 342 & 472 & 1 & \\
\hline 26 & 296 & 102.27257 & 2.2529252 & 342 & 472 & 1 & \\
\hline 27 & 297 & 102.27257 & 2.2529287 & 342 & 472 & 1 & \\
\hline 28 & 298 & 102.27257 & 2.2529323 & 342 & 472 & 1 & \\
\hline 29 & 299 & 102.27257 & 2.2529332 & 342 & 472 & 1 & \\
\hline 30 & 300 & 102.27257 & 2.2529328 & 342 & 472 & 1 & \\
\hline 31 & 301 & 102.27258 & 2.2529326 & 342 & 472 & 1 & \\
\hline 32 & 472 & 102.27258 & 2.252928 & 342 & 472 & 1 & $\begin{array}{c}\text { Antenna } \\
\text { cable }\end{array}$ \\
\hline 33 & 342 & 102.27257 & 2.2528471 & 342 & 384 & 0 & \\
\hline 34 & 208 & 102.27257 & 2.252859 & 342 & 384 & 2 & \\
\hline 35 & 209 & 102.27257 & 2.2528595 & 342 & 384 & 1 & \\
\hline 36 & 210 & 102.27257 & 2.2528599 & 342 & 384 & 1 & \\
\hline 37 & 211 & 102.27256 & 2.2528603 & 342 & 384 & 1 & \\
\hline 38 & 212 & 102.27256 & 2.2528607 & 342 & 384 & 1 & \\
\hline 39 & 213 & 102.27256 & 2.2528615 & 342 & 384 & 1 & \\
\hline 40 & 214 & 102.27256 & 2.2528757 & 342 & 384 & 2 & \\
\hline 41 & 215 & 102.27256 & 2.2528774 & 342 & 384 & 1 & \\
\hline 42 & 232 & 102.27256 & 2.2528798 & 342 & 384 & 1 & \\
\hline 43 & 384 & 102.27256 & 2.2528801 & 342 & 384 & 1 & Bumper \\
\hline 44 & 342 & 102.27257 & 2.2528471 & 342 & 713 & 0 & \\
\hline 45 & 208 & 102.27257 & 2.252859 & 342 & 713 & 2 & \\
\hline 46 & 207 & 102.27258 & 2.2528588 & 342 & 713 & 1 & \\
\hline 47 & 206 & 102.27258 & 2.2528583 & 342 & 713 & 1 & \\
\hline 48 & 205 & 102.27258 & 2.252858 & 342 & 713 & 1 & \\
\hline 49 & 204 & 102.27259 & 2.2528575 & 342 & 713 & 1 & \\
\hline 50 & 203 & 102.27259 & 2.2528572 & 342 & 713 & 1 & \\
\hline 51 & 202 & 102.27259 & 2.2528567 & 342 & 713 & 1 & \\
\hline 52 & 201 & 102.27259 & 2.2528565 & 342 & 713 & 1 & \\
\hline 53 & 200 & 102.2726 & 2.252856 & 342 & 713 & 1 & \\
\hline 54 & 199 & 102.2726 & 2.2528555 & 342 & 713 & 1 & \\
\hline 55 & 198 & 102.2726 & 2.2528553 & 342 & 713 & 1 & \\
\hline 56 & 197 & 102.27261 & 2.2528548 & 342 & 713 & 1 & \\
\hline 57 & 196 & 102.27261 & 2.2528543 & 342 & 713 & 1 & \\
\hline 58 & 195 & 102.27261 & 2.2528706 & 342 & 713 & 2 & \\
\hline 59 & 194 & 102.27262 & 2.2528847 & 342 & 713 & 2 & \\
\hline 60 & 193 & 102.27262 & 2.2528996 & 342 & 713 & 2 & \\
\hline 61 & 192 & 102.27262 & 2.2529086 & 342 & 713 & 1 & \\
\hline 62 & 166 & 102.27264 & 2.2529071 & 342 & 713 & 2 & \\
\hline 63 & 165 & 102.27265 & 2.2529057 & 342 & 713 & 2 & \\
\hline 64 & 119 & 102.27265 & 2.2529111 & 342 & 713 & 1 & \\
\hline 65 & 120 & 102.27265 & 2.2529138 & 342 & 713 & 1 & \\
\hline 66 & 121 & 102.27265 & 2.2529178 & 342 & 713 & 1 & \\
\hline 67 & 122 & 102.27265 & 2.2529216 & 342 & 713 & 1 & \\
\hline 68 & 123 & 102.27266 & 2.2529251 & 342 & 713 & 1 & \\
\hline 69 & 124 & 102.27266 & 2.2529265 & 342 & 713 & 1 & \\
\hline 70 & 141 & 102.27266 & 2.2529289 & 342 & 713 & 1 & \\
\hline 71 & 142 & 102.27266 & 2.2529325 & 342 & 713 & 1 & \\
\hline 72 & 143 & 102.27266 & 2.2529367 & 342 & 713 & 1 & \\
\hline 73 & 144 & 102.27266 & 2.25294 & 342 & 713 & 1 & \\
\hline 74 & 145 & 102.27266 & 2.2529414 & 342 & 713 & 1 & \\
\hline 75 & 146 & 102.27266 & 2.2529411 & 342 & 713 & 1 & \\
\hline 76 & 147 & 102.27267 & 2.2529409 & 342 & 713 & 1 & \\
\hline 77 & 148 & 102.27267 & 2.2529407 & 342 & 713 & 1 & \\
\hline 78 & 149 & 102.27267 & 2.2529405 & 342 & 713 & 1 & \\
\hline 79 & 150 & 102.27267 & 2.2529404 & 342 & 713 & 1 & \\
\hline 80 & 151 & 102.27268 & 2.2529402 & 342 & 713 & 1 & \\
\hline 81 & 152 & 102.27268 & 2.25294 & 342 & 713 & 1 & \\
\hline
\end{tabular}


Table A2. Cont.

\begin{tabular}{|c|c|c|c|c|c|c|c|}
\hline SL No. & $\begin{array}{l}\text { Visited } \\
\text { Node }\end{array}$ & Longitude & Latitude & Source Node & $\begin{array}{c}\text { Destination } \\
\text { Node }\end{array}$ & Weight & Item Name \\
\hline 82 & 713 & 102.27268 & 2.2529351 & 342 & 713 & 1 & $\begin{array}{c}\text { Adjusting } \\
\text { mechanism } \\
\text { (adjuster star } \\
\text { wheel) }\end{array}$ \\
\hline 83 & 342 & 102.27257 & 2.2528471 & 342 & 683 & 0 & \\
\hline 84 & 208 & 102.27257 & 2.252859 & 342 & 683 & 2 & \\
\hline 85 & 207 & 102.27258 & 2.2528588 & 342 & 683 & 1 & \\
\hline 86 & 206 & 102.27258 & 2.2528583 & 342 & 683 & 1 & \\
\hline 87 & 205 & 102.27258 & 2.252858 & 342 & 683 & 1 & \\
\hline 88 & 204 & 102.27259 & 2.2528575 & 342 & 683 & 1 & \\
\hline 89 & 203 & 102.27259 & 2.2528572 & 342 & 683 & 1 & \\
\hline 90 & 202 & 102.27259 & 2.2528567 & 342 & 683 & 1 & \\
\hline 91 & 201 & 102.27259 & 2.2528565 & 342 & 683 & 1 & \\
\hline 92 & 200 & 102.2726 & 2.252856 & 342 & 683 & 1 & \\
\hline 93 & 199 & 102.2726 & 2.2528555 & 342 & 683 & 1 & \\
\hline 94 & 198 & 102.2726 & 2.2528553 & 342 & 683 & 1 & \\
\hline 95 & 197 & 102.27261 & 2.2528548 & 342 & 683 & 1 & \\
\hline 96 & 196 & 102.27261 & 2.2528543 & 342 & 683 & 1 & \\
\hline 97 & 195 & 102.27261 & 2.2528706 & 342 & 683 & 2 & \\
\hline 98 & 194 & 102.27262 & 2.2528847 & 342 & 683 & 2 & \\
\hline 99 & 193 & 102.27262 & 2.2528996 & 342 & 683 & 2 & \\
\hline 100 & 192 & 102.27262 & 2.2529086 & 342 & 683 & 1 & \\
\hline 101 & 166 & 102.27264 & 2.2529071 & 342 & 683 & 2 & \\
\hline 102 & 167 & 102.27264 & 2.2529153 & 342 & 683 & 1 & \\
\hline 103 & 168 & 102.27264 & 2.252919 & 342 & 683 & 1 & \\
\hline 104 & 169 & 102.27264 & 2.2529226 & 342 & 683 & 1 & \\
\hline 105 & 170 & 102.27264 & 2.2529264 & 342 & 683 & 1 & \\
\hline 106 & 171 & 102.27264 & 2.2529298 & 342 & 683 & 1 & \\
\hline 107 & 172 & 102.27264 & 2.2529338 & 342 & 683 & 1 & \\
\hline 108 & 173 & 102.27264 & 2.2529376 & 342 & 683 & 1 & \\
\hline 109 & 174 & 102.27264 & 2.2529413 & 342 & 683 & 1 & \\
\hline 110 & 175 & 102.27264 & 2.2529451 & 342 & 683 & 1 & \\
\hline 111 & 176 & 102.27264 & 2.2529486 & 342 & 683 & 1 & \\
\hline 112 & 177 & 102.27264 & 2.2529524 & 342 & 683 & 1 & \\
\hline 113 & 23 & 102.27265 & 2.2529578 & 342 & 683 & 1 & \\
\hline 114 & 24 & 102.27266 & 2.2529569 & 342 & 683 & 2 & \\
\hline 115 & 25 & 102.27266 & 2.2529567 & 342 & 683 & 1 & \\
\hline 116 & 26 & 102.27267 & 2.2529567 & 342 & 683 & 1 & \\
\hline 117 & 27 & 102.27267 & 2.2529564 & 342 & 683 & 1 & \\
\hline 118 & 28 & 102.27267 & 2.2529563 & 342 & 683 & 1 & \\
\hline 119 & 29 & 102.27268 & 2.2529562 & 342 & 683 & 1 & \\
\hline 120 & 30 & 102.27268 & 2.2529559 & 342 & 683 & 1 & \\
\hline 121 & 31 & 102.27268 & 2.2529558 & 342 & 683 & 1 & \\
\hline 122 & 32 & 102.27269 & 2.2529557 & 342 & 683 & 1 & \\
\hline 123 & 33 & 102.27269 & 2.2529554 & 342 & 683 & 1 & \\
\hline 124 & 683 & 102.27269 & 2.2529501 & 342 & 683 & 1 & Bucket seat \\
\hline
\end{tabular}

Total visited nodes $=124$, total cost $=136$.

Table A3. Simulation detail data for car spare parts scenario using MDMSMD for four items search.

\begin{tabular}{cccccccc}
\hline SL No. & $\begin{array}{c}\text { Visited } \\
\text { Node }\end{array}$ & Longitude & Latitude & $\begin{array}{c}\text { Source } \\
\text { Node }\end{array}$ & $\begin{array}{c}\text { Destination } \\
\text { Node }\end{array}$ & Weight & Item Name \\
\hline 1 & 342 & 102.27257 & 2.2528471 & 342 & 472 & 0 & 1 st source \\
2 & 208 & 102.27257 & 2.252859 & 342 & 472 & 2 & \\
3 & 209 & 102.27257 & 2.2528595 & 342 & 472 & 1 & \\
4 & 210 & 102.27257 & 2.2528599 & 342 & 472 & 1 & \\
5 & 211 & 102.27256 & 2.2528603 & 342 & 472 & 1 & \\
\hline
\end{tabular}


Table A3. Cont.

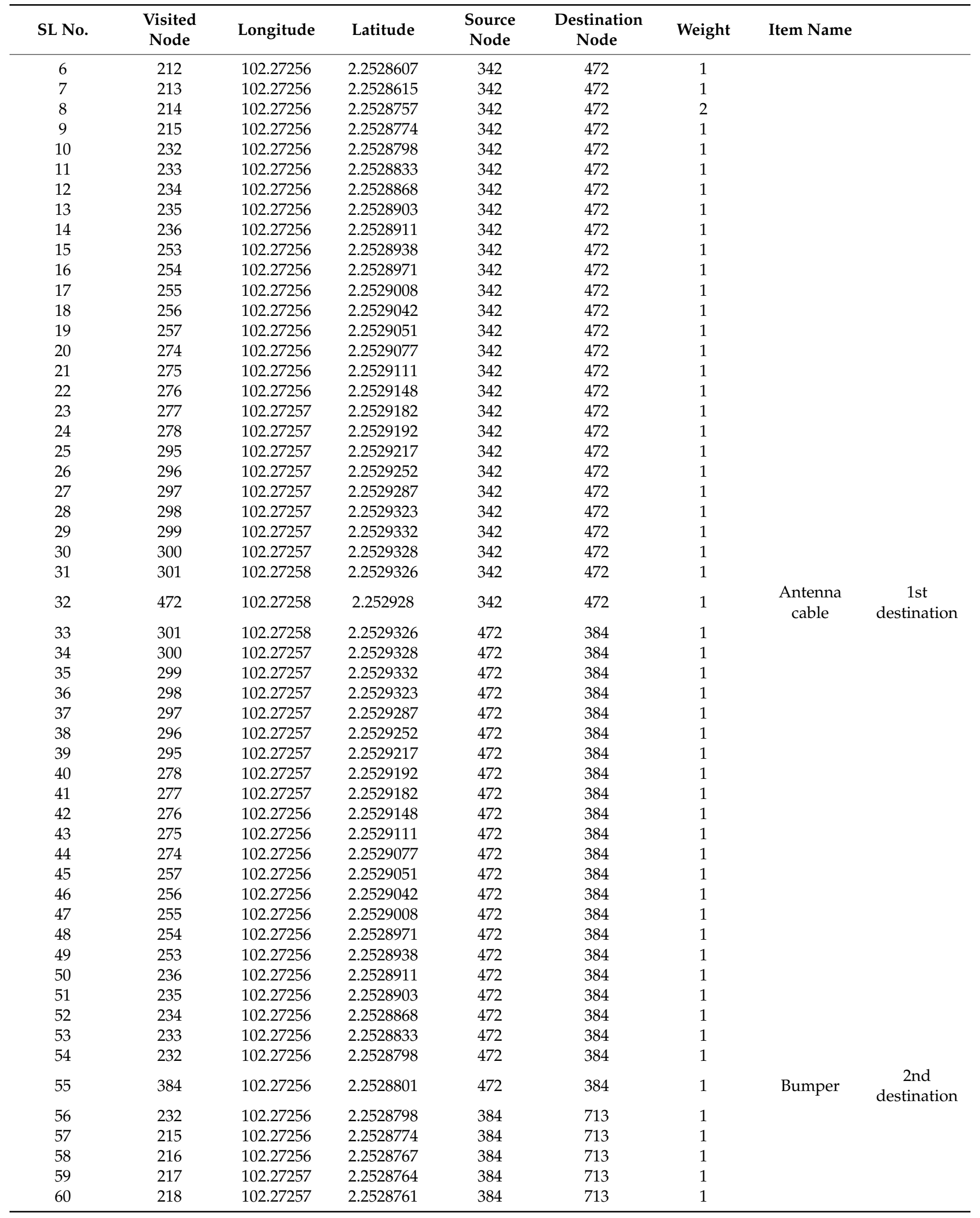


Table A3. Cont.

\begin{tabular}{|c|c|c|c|c|c|c|c|c|}
\hline SL No. & $\begin{array}{l}\text { Visited } \\
\text { Node }\end{array}$ & Longitude & Latitude & $\begin{array}{l}\text { Source } \\
\text { Node }\end{array}$ & $\begin{array}{l}\text { Destination } \\
\text { Node }\end{array}$ & Weight & Item Name & \\
\hline 61 & 219 & 102.27257 & 2.2528755 & 384 & 713 & 1 & & \\
\hline 62 & 220 & 102.27258 & 2.2528752 & 384 & 713 & 1 & & \\
\hline 63 & 221 & 102.27258 & 2.2528749 & 384 & 713 & 1 & & \\
\hline 64 & 222 & 102.27258 & 2.2528744 & 384 & 713 & 1 & & \\
\hline 65 & 223 & 102.27259 & 2.2528741 & 384 & 713 & 1 & & \\
\hline 66 & 224 & 102.27259 & 2.2528738 & 384 & 713 & 1 & & \\
\hline 67 & 225 & 102.27259 & 2.2528735 & 384 & 713 & 1 & & \\
\hline 68 & 226 & 102.27259 & 2.252873 & 384 & 713 & 1 & & \\
\hline 69 & 227 & 102.2726 & 2.2528727 & 384 & 713 & 1 & & \\
\hline 70 & 228 & 102.2726 & 2.2528722 & 384 & 713 & 1 & & \\
\hline 71 & 229 & 102.2726 & 2.2528718 & 384 & 713 & 1 & & \\
\hline 72 & 230 & 102.27261 & 2.2528715 & 384 & 713 & 1 & & \\
\hline 73 & 231 & 102.27261 & 2.2528712 & 384 & 713 & 1 & & \\
\hline 74 & 195 & 102.27261 & 2.2528706 & 384 & 713 & 1 & & \\
\hline 75 & 194 & 102.27262 & 2.2528847 & 384 & 713 & 2 & & \\
\hline 76 & 193 & 102.27262 & 2.2528996 & 384 & 713 & 2 & & \\
\hline 77 & 192 & 102.27262 & 2.2529086 & 384 & 713 & 1 & & \\
\hline 78 & 166 & 102.27264 & 2.2529071 & 384 & 713 & 2 & & \\
\hline 79 & 165 & 102.27265 & 2.2529057 & 384 & 713 & 2 & & \\
\hline 80 & 119 & 102.27265 & 2.2529111 & 384 & 713 & 1 & & \\
\hline 81 & 120 & 102.27265 & 2.2529138 & 384 & 713 & 1 & & \\
\hline 82 & 121 & 102.27265 & 2.2529178 & 384 & 713 & 1 & & \\
\hline 83 & 122 & 102.27265 & 2.2529216 & 384 & 713 & 1 & & \\
\hline 84 & 123 & 102.27266 & 2.2529251 & 384 & 713 & 1 & & \\
\hline 85 & 124 & 102.27266 & 2.2529265 & 384 & 713 & 1 & & \\
\hline 86 & 141 & 102.27266 & 2.2529289 & 384 & 713 & 1 & & \\
\hline 87 & 142 & 102.27266 & 2.2529325 & 384 & 713 & 1 & & \\
\hline 88 & 143 & 102.27266 & 2.2529367 & 384 & 713 & 1 & & \\
\hline 89 & 144 & 102.27266 & 2.25294 & 384 & 713 & 1 & & \\
\hline 90 & 145 & 102.27266 & 2.2529414 & 384 & 713 & 1 & & \\
\hline 91 & 146 & 102.27266 & 2.2529411 & 384 & 713 & 1 & & \\
\hline 92 & 147 & 102.27267 & 2.2529409 & 384 & 713 & 1 & & \\
\hline 93 & 148 & 102.27267 & 2.2529407 & 384 & 713 & 1 & & \\
\hline 94 & 149 & 102.27267 & 2.2529405 & 384 & 713 & 1 & & \\
\hline 95 & 150 & 102.27267 & 2.2529404 & 384 & 713 & 1 & & \\
\hline 96 & 151 & 102.27268 & 2.2529402 & 384 & 713 & 1 & & \\
\hline 97 & 152 & 102.27268 & 2.25294 & 384 & 713 & 1 & & \\
\hline 98 & 713 & 102.27268 & 2.2529351 & 384 & 713 & 1 & $\begin{array}{l}\text { Adjusting } \\
\text { mechanism } \\
\text { (adjuster } \\
\text { star wheel) }\end{array}$ & $\begin{array}{c}\text { 3rd } \\
\text { destination }\end{array}$ \\
\hline 99 & 152 & 102.27268 & 2.25294 & 713 & 683 & 1 & & \\
\hline 100 & 151 & 102.27268 & 2.2529402 & 713 & 683 & 1 & & \\
\hline 101 & 150 & 102.27267 & 2.2529404 & 713 & 683 & 1 & & \\
\hline 102 & 149 & 102.27267 & 2.2529405 & 713 & 683 & 1 & & \\
\hline 103 & 148 & 102.27267 & 2.2529407 & 713 & 683 & 1 & & \\
\hline 104 & 147 & 102.27267 & 2.2529409 & 713 & 683 & 1 & & \\
\hline 105 & 146 & 102.27266 & 2.2529411 & 713 & 683 & 1 & & \\
\hline 106 & 145 & 102.27266 & 2.2529414 & 713 & 683 & 1 & & \\
\hline 107 & 162 & 102.27266 & 2.252944 & 713 & 683 & 1 & & \\
\hline 108 & 163 & 102.27266 & 2.2529477 & 713 & 683 & 1 & & \\
\hline 109 & 164 & 102.27266 & 2.2529515 & 713 & 683 & 1 & & \\
\hline 110 & 24 & 102.27266 & 2.2529569 & 713 & 683 & 1 & & \\
\hline 111 & 25 & 102.27266 & 2.2529567 & 713 & 683 & 1 & & \\
\hline 112 & 26 & 102.27267 & 2.2529567 & 713 & 683 & 1 & & \\
\hline 113 & 27 & 102.27267 & 2.2529564 & 713 & 683 & 1 & & \\
\hline 114 & 28 & 102.27267 & 2.2529563 & 713 & 683 & 1 & & \\
\hline
\end{tabular}


Table A3. Cont.

\begin{tabular}{cccccccc}
\hline SL No. & $\begin{array}{c}\text { Visited } \\
\text { Node }\end{array}$ & Longitude & Latitude & $\begin{array}{c}\text { Source } \\
\text { Node }\end{array}$ & $\begin{array}{c}\text { Destination } \\
\text { Node }\end{array}$ & Weight & Item Name \\
\hline 115 & 29 & 102.27268 & 2.2529562 & 713 & 683 & 1 & \\
116 & 30 & 102.27268 & 2.2529559 & 713 & 683 & 1 & \\
117 & 31 & 102.27268 & 2.2529558 & 713 & 683 & 1 & \\
118 & 32 & 102.27269 & 2.2529557 & 713 & 683 & 1 & \\
119 & 33 & 102.27269 & 2.2529554 & 713 & 683 & 1 & Bucket seat destination \\
120 & 683 & 102.27269 & 2.2529501 & 713 & 683 & 1 & dis \\
\hline
\end{tabular}

Total visited nodes $=120$, total $\operatorname{cost}=125$.

\section{References}

1. Adamatzky, A. Shortest Path Solvers. From Software to Wetware; Springer: Berlin/Heidelberg, Germany, 2018 ; Volume 32.

2. Papadopoulos, S.; Kompatsiaris, Y.; Vakali, A.; Spyridonos, P. Community detection in social media, performance and application considerations. J. Data Min. Knowl. Discov. 2012, 24, 515-554. [CrossRef]

3. Kwon, Y.S.; Sohn, M.Y. Classification of Efficient Total Domination Sets of Circulant Graphs of Degree 5. Symmetry 2020, 12, 1944. [CrossRef]

4. Yang, L.; Li, D.; Tan, R. Shortest Path Solution of Trapezoidal Fuzzy Neutrosophic Graph Based on Circle-Breaking Algorithm. Symmetry 2020, 12, 1360. [CrossRef]

5. Kalaitzakis, A. Comparative Study of Community Detection Algorithms in Social Networks. Ph.D. Thesis, Technological Educational Institute of Crete, Heraklion, Greece, 1939.

6. Bharath-Kumar, K.; Jaffe, J.M. Routing to Multiple Destinations in Computer Networks. IEEE Trans. Commun. 1983, 31, 343-351. [CrossRef]

7. Ben Ticha, H.; Absi, N. A Solution Method for the Multi-Destination Bi-Objectives Shortest Path Problem; Elsevier: Amsterdam, The Netherlands, 2017. [CrossRef]

8. Dong, Y.F.; Xia, H.M.; Zhou, Y.C. Disordered and Multiple Destinations Path Planning Methods for Mobile Robot in Dynamic Environment. J. Electr. Comput. Eng. 2016, 2016, 3620895. [CrossRef]

9. Sepehrifar, M.K.; Zamanifar, K.; Sepehrifar, M.B. An Algorithm to Select the Optimal Composition of the Services. J. Theor. Appl. Inf. Technol. 2009, 8, 154-161.

10. Wang, W.; Uehara, M.; Ozaki, H. Evaluation of navigation based on system optimal traffic assignment for connected cars. Int. J. Grid Util. Comput. 2020, 11, 525-532. [CrossRef]

11. Burgaña, J.L. Design and evaluation of a link-state routing protocol for Internet-Wide Geocasting. Master's Thesis, University of Twente, Enschede, The Netherlands, 2017.

12. Sepehrifar, M.K.; Fanian, A.; Sepehrifar, B. Shortest Path Computation in a Network with Multiple Destinations. Arab. J. Sci. Eng. 2020, 45, 3223-3231. [CrossRef]

13. Jubair, F.; Hawa, M. Exploiting Obstacle Geometry to Reduce Search Time in Grid-Based Pathfinding. Symmetry 2020, 12, 1186. [CrossRef]

14. Rahman, M.S.; Ahmed, S. A survey on pairwise compatibility graphs. AKCE Int. J. Graphs Comb. 2020, 17, 788-795. [CrossRef]

15. Easttom, W.; Adda, M. An enhanced view of incidence functions for applying graph theory to modeling network intrusions. WSEAS Trans. Inf. Sci. Appl. 2020, 15, 102-109.

16. Cormen, T.H.; Leiserson, C.E.; Rivest, R.L.; Stein, C. Introduction to Algorithms; MIT Press: Cambridge, MA, USA, 2009.

17. Arman, N.; Khamayseh, F. A Path-Compression Approach for Improving Shortest-Path Algorithms. Int. J. Electr. Comput. Eng. 2015, 5, 772-781. [CrossRef]

18. Hakeem, A.; Gehani, N.; Ding, X.; Curtmola, R.; Borcea, C. Multi-destination vehicular route planning with parking and traffic constraints. In Proceedings of the 16th EAI International Conference on Mobile and Ubiquitous Systems: Computing, Networking and Services, Houston, TX, USA, 12-14 November 2019; pp. 298-307.

19. Hu, W.-C.; Wu, H.-T.; Cho, H.-H.; Tseng, F.-H. Optimal Route Planning System for Logistics Vehicles Based on Artificial Intelligence. J. Internet Technol. 2020, 21, 757-764.

20. Li, X.; Yin, H. Optimal Mobile Relays Positions and Resource Allocation for Multi-Relay Multi-Destination Wireless Networks. IEEE Access 2020, 8, 47993-48004. [CrossRef]

21. Anđelić, M.; Živković, D. Efficient Algorithm for Generating Maximal L-Reflexive Trees. Symmetry 2020, 12, 809. [CrossRef]

22. Zhang, H.; Zhang, Z. AOA-Based Three-Dimensional Positioning and Tracking Using the Factor Graph Technique. Symmetry 2020, 12, 1400. [CrossRef]

23. Panić, B.; Kontrec, N.; Vujošević, M.; Panić, S. A Novel Approach for Determination of Reliability of Covering a Node from K Nodes. Symmetry 2020, 12, 1461. [CrossRef]

24. Slamin, S.; Adiwijaya, N.O.; Hasan, M.A.; Dafik, D.; Wijaya, K. Local Super Antimagic Total Labeling for Vertex Coloring of Graphs. Symmetry 2020, 12, 1843. [CrossRef] 
25. Martínez, A.C.; García, S.C.; García, A.C.; Del Rio, A.M.G. On the Outer-Independent Roman Domination in Graphs. Symmetry 2020, 12, 1846. [CrossRef]

26. Martínez, A.C.; Estrada-Moreno, A.; Rodríguez-Velázquez, J.A. Secure w-Domination in Graphs. Symmetry 2020, 12, 1948. [CrossRef]

27. Lv, Y.; Liu, M.; Xiang, Y. Fast Searching Density Peak Clustering Algorithm Based on Shared Nearest Neighbor and Adaptive Clustering Center. Symmetry 2020, 12, 2014. [CrossRef]

28. Balakrishnan, A.; Banciu, M.; Glowacka, K.; Mirchandani, P. Hierarchical approach for survivable network design. Eur. J. Oper. Res. 2013, 225, 223-235. [CrossRef]

29. Fredman, M.L.; Tarjan, R.E. Fibonacci heaps and their uses in improved network optimization algorithms. J. ACM 1987, 34, 596-615. [CrossRef]

30. Qu, T.; Cai, Z. A Fast Isomap Algorithm Based on Fibonacci Heap. In International Conference in Swarm Intelligence; Springer: Cham, Switzerland, 2015; pp. 225-231.

31. Lu, X.; Camitz, M. Finding the shortest paths by node combination. Appl. Math. Comput. 2011, 217, 6401-6408. [CrossRef]

32. Orlin, J.B.; Madduri, K.; Subramani, K.; Williamson, M. A faster algorithm for the single source shortest path problem with few distinct positive lengths. J. Discret. Algorithms 2010, 8, 189-198. [CrossRef]

33. Thorup, M. Undirected single-source shortest paths with positive integer weights in linear time. J. ACM 1999, 46, 362-394. [CrossRef]

34. Thorup, M. On RAM Priority Queues. SIAM J. Comput. 2000, 30, 86-109. [CrossRef]

35. MacCormick, J. What Can Be Computed? A Practical Guide to the Theory of Computation; Princeton University Press: Princeton, NJ, USA, 2018.

36. Xu, M.; Liu, Y.; Huang, Q.; Zhang, Y.; Luan, G. An improved Dijkstra's shortest path algorithm for sparse network. Appl. Math. Comput. 2007, 185, 247-254. [CrossRef]

37. Holzer, M.; Schulz, F.; Wagner, D.; Willhalm, T. Combining speed-up techniques for shortest-path computations. ACM J. Exp. Algorithmics 2005, 10, 2-5. [CrossRef]

38. Chen, Y.-Z.; Shen, S.-F.; Chen, T.; Yang, R. Path Optimization Study for Vehicles Evacuation based on Dijkstra Algorithm. Procedia Eng. 2014, 71, 159-165. [CrossRef]

39. Madkour, A.; Aref, W.G.; Rehman, F.U.; Rahman, M.A.; Basalamah, S. A survey of shortest-path algorithms. arXiv 2017, arXiv:1705.02044.

40. Okengwu, U.A.; Nwachukwu, E.O.; Osegi, E.N. Modified Dijkstra algorithm with invention hierarchies applied to a conic graph. arXiv 2015, arXiv:1503.02517.

41. Hong, Y.; Li, D.; Wu, Q.; Xu, H. Priority-Oriented Route Network Planning for Evacuation in Constrained Space Scenarios. J. Optim. Theory Appl. 2019, 181, 279-297. [CrossRef]

42. Jin, W.; Chen, S.; Jiang, H. Finding the K shortest paths in a time-schedule network with constraints on arcs. Comput. Oper. Res. 2013, 40, 2975-2982. [CrossRef]

43. Ananta, M.T.; Jiang, J.-R.; Muslim, M.A. Multicasting with the extended Dijkstra's shortest path algorithm for software defined networking. Int. J. Appl. Eng. Res. 2014, 9, 21017-21030. 